Алгебра и анализ

Toм 20 (2008), № 5
St. Petersburg Math. J.

Vol. 20 (2009), No. 5, Pages 709-736

S 1061-0022(09)01069-3

Article electronically published on July 21, 2009

\title{
COMPLEXITY OF A STANDARD BASIS OF A D-MODULE
}

\author{
D. YU. GRIGORIEV AND A. L. CHISTOV
}

\begin{abstract}
A double-exponential upper bound is obtained for the degree and for the complexity of constructing a standard basis of a $D$-module. This generalizes a well-known bound for the complexity of a Gröbner basis of a module over the algebra of polynomials. It should be emphasized that the bound obtained cannot be deduced immediately from the commutative case. To get the bound in question, a new technique is developed for constructing all the solutions of a linear system over a homogeneous version of a Weyl algebra.
\end{abstract}

\section{INTRODUCTION}

Let $A$ be the Weyl algebra $F\left[X_{1}, \ldots, X_{n}, \frac{\partial}{\partial X_{1}}, \ldots, \frac{\partial}{\partial X_{n}}\right]$ (or the algebra of differential operators $\left.F\left(X_{1}, \ldots, X_{n}\right)\left[\frac{\partial}{\partial X_{1}}, \ldots, \frac{\partial}{\partial X_{n}}\right]\right)$. For brevity, we denote $D_{i}=\frac{\partial}{\partial X_{i}}, 1 \leq i \leq n$. Any $A$-module is called a $D$-module. It is well known that an $A$-module that is a submodule of a free finitely generated $A$-module has a Janet basis (if $A$ is a Weyl algebra, it is often called a standard basis, but in this paper it is natural and convenient to call it a Janet basis also in that case). Historically, it was first introduced in 9. In the more recent time of developing computer algebra, Janet bases were studied in [5, 14, 10, The Janet bases generalize the Gröbner bases, which were widely used in the algebra of polynomials (see, e.g., 3]). For the Gröbner bases, a double-exponential complexity bound was obtained in [12, 6] with the help of [1. Later, sharper results on the same subject (with independent and self-contained proofs) were obtained in [4.

Surprisingly, no complexity bound for Janet bases has been established so far. The reason is clear: the problem is not easy. In the present paper we fill this very essential gap and prove a double-exponential upper bound for complexity. On the other hand, a double-exponential complexity lower bound for Gröbner bases [12, 15] provides by the same token a bound for Janet bases.

Notice also that there has been a folklore opinion that the problem of constructing a Janet basis reduces easily to the commutative case by considering the associated graded module, and, on the other hand, in the commutative case [6, 12, 4, the doubleexponential upper bound is well known. But this turns out to be a fallacy! From a known system of generators of a D-module, no system of generators (even not necessarily a Gröbner basis) of the associated graded module can be obtained immediately. The main problem here is to construct such a system of generators of the graded module. It may have elements of degrees $(d l)^{2^{O(n)}}$; see the notation below. Then, indeed, to the last system of generators of large degrees, one can apply the result known in the commutative case and get the bound $\left((d l)^{2^{O(n)}}\right)^{2^{O(n)}}=(d l)^{2^{O(n)}}$. Thus, some new ideas specific to the noncommutative case are needed.

2000 Mathematics Subject Classification. Primary 16Z05.

Key words and phrases. Weyl algebra, Janet basis, Gröbner basis. 
We are interested in estimates for Janet bases of $A$-submodules of $A^{l}$. A Janet basis depends on the choice of a linear order on the monomials (we define them also for $l>1$ ). In this paper we consider the most general linear orders on the monomials in $A^{l}$. They satisfy conditions (a) and (b) in $\S 1$ and are said to be admissible. If additionally a linear order satisfies condition (c) in $\S 1$, then it is said to be degree-compatible. For any admissible linear order, the reduced Janet basis is chosen canonically and it is defined uniquely; see $\S 1$. We prove the following result.

Theorem 1. For any real number $d \geq 2$ and any admissible linear order on the monomials in $A^{l}$, any left $A$-submodule $I$ of $A^{l}$ generated by elements of degree less than $d$ (with respect to the filtration in the corresponding algebra; see $\S \S 1$ and 9) has a Janet basis with the degrees and the number of its elements less than

$$
(d l)^{2^{O(n)}} \text {. }
$$

The same upper estimate (1) is valid for the number of elements of the reduced Janet basis of the module I with respect to the linear order in question on the monomials.

If, moreover, this linear order is degree-compatible or it is an arbitrary admissible order, but $l=1$, then also the degrees of all the elements of the reduced Janet basis of the module I are bounded from above by (1).

We prove this theorem in detail for the case of the Weyl algebra $A$. The proof for the case of the algebra of differential operators is similar. It is sketched in $\S 9$. Theorem 1 implies that the Hilbert function $H(I, m)$, see $\S 1$, of the $A$-submodule from this theorem is stable for $m \geq(d l)^{2^{O(n)}}$ and that the absolute values of all coefficients of the Hilbert polynomial of $I$ are bounded from above by $(d l)^{2^{O(n)}}$; cf., e.g., 12. This fact follows directly from (11), Lemma 12 in Appendix 1, Lemma 2, and Theorem 2. We mention that, in [7, a similar bound was established for the leading coefficient of the Hilbert polynomial.

Now we outline the plan of our proof of Theorem 1. Below, the first occurrences of some terms introduced in the paper are italicized. The main tool in the proof is a homogenized Weyl algebra ${ }^{h} A$ (or respectively, a homogenized algebra of differential operators ${ }^{h} B$ ). It is introduced in $\S 3$ (respectively, in $\S 9$ ). The algebra ${ }^{h} A$ (respectively, ${ }^{h} B$ ) is generated over the ground field $F$ by $X_{0}, \ldots, X_{n}, D_{1}, \ldots, D_{n}$ (respectively, over the field $F\left(X_{1}, \ldots, X_{n}\right)$ by $\left.X_{0}, D_{1}, \ldots, D_{n}\right)$. Here $X_{0}$ is a new homogenizing variable. In the algebra ${ }^{h} A$ (respectively, ${ }^{h} B$ ), relations (13) in $\S 3$ (respectively, (54) in $\S 9$ ) hold true for these generators.

We define the homogenization ${ }^{h} I$ of the module $I$. It is an ${ }^{h} A$-submodule of ${ }^{h} A^{l}$. The main problem is to estimate the degrees of a system of generators of ${ }^{h} I$. These estimates are central to the paper. They are deduced from Theorem 2 in $\S 7$. That theorem is devoted to the problem of solving systems of linear equations over the ring ${ }^{h} A$; we discuss this below in more detail.

The system of generators of ${ }^{h} I$ gives a system of generators of the graded $\operatorname{gr}(A)$-module $\operatorname{gr}(I)$ corresponding to $I$. But $\operatorname{gr}(A)$ is a polynomial ring. Hence, using Lemma 12 in Appendix 1, we get a double-exponential bound $(d l)^{2^{O(n)}}$ for the stabilization of the Hilbert function of $\operatorname{gr}(I)$ and for the absolute values of the coefficients of the Hilbert polynomial of $\operatorname{gr}(I)$. Therefore, there is a similar bound for the stabilization of the Hilbert functions of $I$ and the coefficients of the Hilbert polynomial of $I$; see $\S 2$.

But the Hilbert functions of the modules $I$ and ${ }^{h} I$ coincide; see $\S 3$. Hence, the last bound serves also for the stabilization of the Hilbert functions of ${ }^{h} I$ and the coefficients of the Hilbert polynomial of ${ }^{h} I$. In $\S 5$ we introduce the linear order on the monomials in ${ }^{h} A^{l}$ induced by the initial linear order on the monomials in $A^{l}$ (the homogenizing 
variable $X_{0}$ is the least possible in this ordering). Next, we define the Janet basis of ${ }^{h} I$ with respect to the induced linear order on the monomials. Such a basis can be obtained by homogenization of the elements of a Janet basis of $I$ with respect to the initial linear order; see Lemma 3 (iii).

For every element $f \in{ }^{h} A$, denote by $\operatorname{Hdt}(f) \in{ }^{h} A$ the greatest monomial of the element $f$; i.e., each monomial of $f-\operatorname{Hdt}(f)$ is less than $\operatorname{Hdt}(f)$ with respect to the induced linear order on the monomials in ${ }^{h} A$. Let $\operatorname{Hdt}\left({ }^{h} I\right)=\left\{\operatorname{Hdt}(f): f \in{ }^{h} I\right\}$ be the set of all the greatest monomials of the elements of the module ${ }^{h} I$; see $\S 4$. Let ${ }^{c} I \subset{ }^{c} A^{l}$, see $\S 4$, be the module over the polynomial ring ${ }^{c} A=F\left[X_{0}, \ldots, X_{n}, D_{1}, \ldots, D_{n}\right]$ generated by all monomials in $\operatorname{Hdt}\left({ }^{h} I\right)$ (they are viewed now as elements of $\left.{ }^{c} A^{l}\right)$. Then the Hilbert functions of the modules ${ }^{h} I$ and ${ }^{c} I$ coincide. Thus, we have the same double-exponential estimate as above for the stabilization of the Hilbert function of ${ }^{C} I$ and the coefficients of the Hilbert polynomial of ${ }^{c} I$. Now, using Lemma 13, we get the estimate $(d l)^{2^{O(n)}}$ for the monomial system of generators of ${ }^{C} I$. This gives a bound for the degrees of the elements of the reduced Janet basis of ${ }^{h} I$, and hence, by Lemma 11, also the bound from Theorem 1 for the required Janet basis (respectively, in the case where the initial order is degreecompatible, for the reduced Janet basis) of $I$. Estimation of the degrees of the elements of the reduced Janet basis in the case where $l=1$ requires special considerations; see $\S 8$.

Remark 1. The question as to whether there is a double-exponential upper bound for the degrees of the elements of the reduced Janet basis with respect to an arbitrary admissible linear order on monomials in the case where $l>1$ remains open. Note the following description of all admissible linear orders on the monomials in $A^{l}$ : each linear order corresponds to a rooted tree. But we do not need this description in the present paper.

The problem of solving systems of linear equations over the homogenized Weyl algebra is central to this paper; see Theorem 2. It is studied in $\S \S 5-7$. A similar problem over the Weyl algebra (without homogenization) was considered in [7]. The principal idea is to try to extend the well-known method of [8], which was developed for the algebra of polynomials, to the homogenized Weyl algebra. There are two principal difficulties with this approach. The first is that in the method from 8 the use of determinants is essential, which should be avoided when we deal with noncommutative algebras. The second is that a kind of Noether normalization theorem is needed in the current situation. Therefore, the analog of the method of 8 requires choosing the leading elements with the smallest possible order $\operatorname{ord}_{X_{0}}$, where $X_{0}$ is a homogenizing variable; see $\S 3$.

The bound obtained for the degree of a Janet basis implies a similar bound for the complexity of its construction. Indeed, by Corollary 1 (it is formulated for the case of a Weyl algebra, but a similar statement is true for the case of the algebra of differential operators), one can compute the linear space of all the elements $z \in I$ of degrees bounded from above by $(d l)^{2^{O(n)}}$. Hence, by Theorem 1, a Janet basis of $I$ can be computed by solving linear systems over $F$ of size bounded from above by $(d l)^{2^{O(n)}}$ (merely with the help of enumeration of all monomials of degrees at most $(d l)^{2^{O(n)}}$ that are possible elements of $\operatorname{Hdt}(I))$. After that, within time polynomial in $(d l)^{2^{O(n)}}$ and in the size of the input, by solving linear systems over $F$ one can obtain the reduced Janet basis of $I$, provided that the upper bound $(d l)^{2^{O(n)}}$ for the degrees of its elements is known; see Theorem 1.

To make our text self-contained, in Appendix 1 (see Lemma 12) we give a short proof of the double-exponential estimate for stabilization of the Hilbert function of a graded module over a graded polynomial ring. The converse of Lemma 12 is also true; see Lemma 13 in Appendix 1. This fact is essential for us. The proof of Lemma 13 involves 
the classical description of the Hilbert function of a homogeneous ideal in $F\left[X_{0}, \ldots, X_{n}\right]$ in terms of the Macaulay constants $b_{n+2}, \ldots, b_{1}$ and the constant $b_{0}$ introduced in [4]. In Appendix 2, we give an independent and instructive proof of Proposition 1, which is similar to Lemma 13. In a sense, Proposition 1 is even stronger than Lemma 13, because to apply it one does not need a bound for the stabilization of the Hilbert function. Of course, a reference to Proposition 1 can be used in place of Lemma 13 in our paper.

\section{$\S 1$. Definition of a Janet basis}

Let $A=F\left[X_{1}, \ldots, X_{n}, D_{1}, \ldots, D_{n}\right], n \geq 1$, be a Weyl algebra over a field $F$. So, $A$ is determined by the following relations:

(2) $X_{v} X_{w}=X_{w} X_{v}, D_{v} D_{w}=D_{w} D_{v}, D_{v} X_{v}-X_{v} D_{v}=1, X_{v} D_{w}=D_{w} X_{v}, \quad v \neq w$.

By (2), any element $f \in A$ admits a unique representation in the form

$$
f=\sum_{i_{1}, \ldots, i_{n}, j_{1}, \ldots, j_{n} \geq 0} f_{i_{1}, \ldots, i_{n}, j_{1}, \ldots, j_{n}} X_{1}^{i_{1}} \cdots X_{n}^{i_{n}} D_{1}^{j_{1}} \cdots D_{n}^{j_{n}},
$$

where all $f_{i_{1}, \ldots, i_{n}, j_{1}, \ldots, j_{n}}$ belong to $F$ and only a finite number of the $f_{i_{1}, \ldots, i_{n}, j_{1}, \ldots, j_{n}}$ are nonzero. For brevity, we denote $\mathbb{Z}_{+}=\{z \in \mathbb{Z}: z \geq 0\}$ and

$$
\begin{aligned}
i & =\left(i_{1}, \ldots, i_{n}\right), \quad j=\left(j_{1}, \ldots, j_{n}\right), \quad f_{i, j}=f_{i_{1}, \ldots, i_{n}, j_{1}, \ldots, j_{n}}, \\
X^{i} & =X_{1}^{i_{1}} \cdots X_{n}^{i_{n}}, \quad D^{j}=D_{1}^{j_{1}} \cdots D_{n}^{j_{n}}, \quad f=\sum_{i, j} f_{i, j} X^{i} D^{j}, \\
|i| & =i_{1}+\cdots+i_{n}, \quad i+j=\left(i_{1}+j_{1}, \ldots, i_{n}+j_{n}\right) .
\end{aligned}
$$

Thus, $i, j \in \mathbb{Z}_{+}^{n}$ are multi-indices. By definition, the degree of $f$ is

$$
\operatorname{deg} f=\operatorname{deg}_{X_{1}, \ldots, X_{n}, D_{1}, \ldots, D_{n}} f=\max \left\{|i|+|j|: f_{i, j} \neq 0\right\} .
$$

Let $M$ be a left $A$-module given by its generators $m_{1}, \ldots, m_{l}, l \geq 0$, and relations

$$
\sum_{1 \leq w \leq l} a_{v, w} m_{w}, \quad 1 \leq v \leq k
$$

where $k \geq 0$ and all $a_{v, w}$ are in $A$. We assume that $\operatorname{deg} a_{v, w}<d$ for all $v, w$, where $d \geq 2$. By (5), we have the exact sequence

$$
A^{k} \stackrel{\iota}{\rightarrow} A^{l} \stackrel{\pi}{\rightarrow} M \rightarrow 0
$$

of left $A$-modules. Denote $I=\iota\left(A^{k}\right) \subset A^{l}$. If $l=1$, then $I$ is a left ideal of $A$ and $M=A / I$. In the general case, $I$ is generated by the elements

$$
\left(a_{v, 1}, \ldots, a_{v, l}\right) \in A^{l}, \quad 1 \leq v \leq k .
$$

For an integer $m \geq 0$, put

$$
A_{m}=\{a: \operatorname{deg} a \leq m\}, \quad M_{m}=\pi\left(A_{m}^{l}\right), \quad I_{m}=I \cap A_{m}^{l} .
$$

So, now $A, M, I$ are filtered modules with filtrations $A_{m}, M_{m}, I_{m}, m \geq 0$, respectively, and the sequence of homomorphisms of vector spaces

$$
0 \rightarrow I_{m} \rightarrow A_{m}^{l} \rightarrow M_{m} \rightarrow 0
$$

induced by (6) is exact for every $m \geq 0$. The Hilbert function $H(M, m)$ of the module $M$ is defined by the formula

$$
H(M, m)=\operatorname{dim}_{F} M_{m}, \quad m \geq 0 .
$$

Each element of $A^{l}$ can be uniquely represented as an $F$-linear combination of elements $e_{v, i, j}=\left(0, \ldots, 0, X^{i} D^{j}, 0, \ldots, 0\right)$, where $i, j \in \mathbb{Z}_{+}^{n}$ are multi-indices, see (4), and the 
nonzero monomial $X^{i} D^{j}$ is at the position $v, 1 \leq v \leq l$. Thus, every element $f \in A^{l}$ can be represented in the form

$$
f=\sum_{v, i, j} f_{v, i, j} e_{v, i, j}, \quad f_{v, i, j} \in F .
$$

The elements $e_{v, i, j}$ will be called monomials.

Consider a linear order $<$ on the set of all the monomials $e_{v, i, j}$ or, what is the same, on the set of triples $(v, i, j), 1 \leq v \leq l, i, j \in \mathbb{Z}_{+}^{n}$. If $f \neq 0$, we put

$$
o(f)=\max \left\{(v, i, j): f_{v, i, j} \neq 0\right\}
$$

see (8). Set

$$
o(0)=-\infty<o(f)
$$

for every $0 \neq f \in A$. The leading monomial of an element $0 \neq f \in A^{l}$ is defined by the formula

$$
\operatorname{Hdt}(f)=f_{v, i, j} e_{v, i, j}
$$

where $o(f)=(v, i, j)$. Put $\operatorname{Hdt}(0)=0$. Hence, $o(f-\operatorname{Hdt}(f))<o(f)$ if $f \neq 0$. For $f_{1}, f_{2} \in A^{l}$, if $o\left(f_{1}\right)<o\left(f_{2}\right)$, we shall write $f_{1}<f_{2}$. We shall require additionally that

(a) for all multi-indices $i, j, i^{\prime}, j^{\prime}$ and all $1 \leq v \leq l$, if $i_{1} \leq i_{1}^{\prime}, \ldots, i_{n} \leq i_{n}^{\prime}$ and $j_{1} \leq j_{1}^{\prime}, \ldots, j_{n} \leq j_{n}^{\prime}$, then $(v, i, j) \leq\left(v, i^{\prime}, j^{\prime}\right)$;

(b) for all multi-indices $i, j, i^{\prime}, j^{\prime}, i^{\prime \prime}, j^{\prime \prime}$ and all $1 \leq v, v^{\prime} \leq l$, if $(v, i, j)<\left(v^{\prime}, i^{\prime}, j^{\prime}\right)$, then $\left(v, i+i^{\prime \prime}, j+j^{\prime \prime}\right)<\left(v^{\prime}, i^{\prime}+i^{\prime \prime}, j^{\prime}+j^{\prime \prime}\right)$.

Conditions (a) and (b) imply that, for all $f_{1}, f_{2} \in A^{l}$ and every nonzero $a \in A$, if $f_{1}<f_{2}$, then $a f_{1}<a f_{2}$; i.e., the linear order under consideration is compatible with the product. Any linear order on the monomials $e_{v, i, j}$ satisfying (a) and (b) will be called admissible. Consider additionally the condition

(c) for all multi-indices $i, j, i^{\prime}, j^{\prime}$ and all $1 \leq v, v^{\prime} \leq l$, if $|i|+|j|<\left|i^{\prime}\right|+\left|j^{\prime}\right|$, then $(v, i, j)<\left(v^{\prime}, i^{\prime}, j^{\prime}\right)$.

Any linear order on the monomials $e_{v, i, j}$ satisfying (c) will be called a degree-compatible order (in what follows all the degree-compatible linear orders that we consider will also be admissible, i.e., satisfying (a) and (b)).

For every subset $E \subset A^{l}$ we put $\operatorname{Hdt}(E)=\{\operatorname{Hdt}(f): f \in E\}$. In particular,

$$
\operatorname{Hdt}(I)=\{\operatorname{Hdt}(f): f \in I\} .
$$

Thus, $\operatorname{Hdt}(I)$ is a subset of $A^{l}$. By definition, a family $f_{1}, \ldots, f_{m}$ of elements of $I$ is a Janet basis of the module $I$ if and only if

1) $\operatorname{Hdt}(I)=\operatorname{Hdt}\left(A f_{1}\right) \cup \cdots \cup \operatorname{Hdt}\left(A f_{m}\right)$.

Next, the Janet basis $f_{1}, \ldots, f_{m}$ of $I$ is reduced if and only if the following conditions are fulfilled.

2) $f_{1}, \ldots, f_{m}$ does not contain a smaller Janet basis of $I$.

3) $\operatorname{Hdt}\left(f_{1}\right)>\cdots>\operatorname{Hdt}\left(f_{m}\right)$.

4) The coefficient from $F$ of every monomial $\operatorname{Hdt}\left(f_{v}\right), 1 \leq \alpha \leq m$, is 1 .

5) Let $f_{\alpha}=\sum_{v, i, j} f_{\alpha, v, i, j} e_{v, i, j}$ be the representation (3) for $f_{\alpha}, 1 \leq \alpha \leq m$. Then for all $1 \leq \alpha<\beta \leq m$, all $1 \leq v \leq l$, and all multi-indices $i, j$, the monomial $f_{\alpha, v, i, j} e_{v, i, j}$ does not belong to $\operatorname{Hdt}\left(A f_{\beta} \backslash\{0\}\right)$.

Let $C$ denote the ring of polynomials in $X_{1}, \ldots, X_{n}, D_{1}, \ldots, D_{n}$ with coefficients in $F$ (we can take $C=\operatorname{gr}(A)$; see the next section). For every $f \in A^{l}$, the monomial $\operatorname{Hdt}(f)$ can be viewed as an element of $C^{l}$. To avoid ambiguity, we denote it by $\operatorname{Hdtc}(f) \in C^{l}$. Now, $f_{1}, \ldots, f_{m}$ is a Janet basis of the module $I$ if and only if the $C$-submodule of $C^{l}$ generated by $\operatorname{Hdtc}\left(f_{\alpha}\right), 1 \leq \alpha \leq m$, contains all the elements $\operatorname{Hdtc}(f), f \in A$. Since the 
ring $C$ is Noetherian, the module $I$ under consideration admits a Janet basis. Moreover, the reduced Janet basis of $I$ is uniquely determined.

\section{$\S 2$. The graded module corresponding to a $D$-module}

Put $A_{v}=I_{v}=M_{v}=0$ for $v<0$ and

$$
\operatorname{gr}(A)=\bigoplus_{m \geq 0} A_{m} / A_{m-1}, \quad \operatorname{gr}(I)=\bigoplus_{m \geq 0} I_{m} / I_{m-1}, \quad \operatorname{gr}(M)=\bigoplus_{m \geq 0} M_{m} / M_{m-1}
$$

The structure algebra on $A$ induces the structure of a graded algebra on $\operatorname{gr}(A)$. Thus, $\operatorname{gr}(A)=F\left[X_{1}, \ldots, X_{n}, D_{1}, \ldots, D_{n}\right]$ is an algebra of polynomials with respect to the variables $X_{1}, \ldots, X_{n}, D_{1}, \ldots, D_{n}$. Next, $\operatorname{gr}(I)$ and $\operatorname{gr}(M)$ are graded $\operatorname{gr}(A)$-modules. Using (7), we get the exact sequences

$$
0 \rightarrow I_{m} / I_{m-1} \rightarrow\left(A_{m} / A_{m-1}\right)^{l} \rightarrow M_{m} / M_{m-1} \rightarrow 0, \quad m \geq 0 .
$$

The Hilbert function of the module $\operatorname{gr}(M)$ is defined as follows:

$$
H(\operatorname{gr}(M), m)=\operatorname{dim}_{F} M_{m} / M_{m-1}, \quad m \geq 0 .
$$

Obviously,

$$
H(M, m)=\sum_{0 \leq v \leq m} H(\operatorname{gr}(M), v), \quad H(\operatorname{gr}(M), m)=H(M, m)-H(M, m-1)
$$

for every $m \geq 0$.

For arbitrary $a \in M$, we denote by $\operatorname{gr}(a) \in \operatorname{gr}(M)$ the image of $a$ in $\operatorname{gr}(M)$.

Lemma 1. Assume that $b_{1}, \ldots, b_{s}$ is a system of generators of $I$. Let $\nu_{i}=\operatorname{deg} b_{i}$, $1 \leq i \leq s$. Suppose that

$$
I_{m}=\left\{\sum_{1 \leq v \leq \mu} c_{v} b_{v}: c_{v} \in A, \operatorname{deg} c_{v} \leq m-\nu_{v}, 1 \leq i \leq s\right\}
$$

for every $m \geq 0$. Then $\operatorname{gr}\left(b_{1}\right), \ldots, \operatorname{gr}\left(b_{s}\right)$ is a system of generators of the $\operatorname{gr}(A)$-module $\operatorname{gr}(I)$.

Proof. This is straightforward.

\section{§3. Homogenization of the Weyl Algebra}

Let $X_{0}$ be a new variable. Consider the algebra ${ }^{h} A=F\left[X_{0}, X_{1}, \ldots, X_{n}, D_{1}, \ldots, D_{n}\right]$ given by the relations

$$
\begin{aligned}
X_{v} X_{w} & =X_{w} X_{v}, \quad D_{v} D_{w}=D_{w} D_{v} \quad \text { for all } v, w \\
D_{v} X_{v}-X_{v} D_{v} & =X_{0}^{2}, \quad 1 \leq v \leq n, \quad X_{v} D_{w}=D_{w} X_{v} \quad \text { for all } \quad v \neq w
\end{aligned}
$$

The algebra ${ }^{h} A$ is Noetherian, like the Weyl algebra $A$. By (13), any element $f \in{ }^{h} A$ can be uniquely represented in the form

$$
f=\sum_{i_{0}, i_{1}, \ldots, i_{n}, j_{1}, \ldots, j_{n} \geq 0} f_{i_{0}, \ldots, i_{n}, j_{1}, \ldots, j_{n}} X_{0}^{i_{0}} \cdots X_{n}^{i_{n}} D_{1}^{j_{1}} \cdots D_{n}^{j_{n}}
$$

where all $f_{i_{0}, \ldots, i_{n}, j_{1}, \ldots, j_{n}}$ are in $F$ and only finitely many of $f_{i_{0}, \ldots, i_{n}, j_{1}, \ldots, j_{n}}$ are nonzero. Let $i, j$ be multi-indices; see (4). Denote for brevity

$$
\begin{aligned}
i & =\left(i_{1}, \ldots, i_{n}\right), \quad j=\left(j_{1}, \ldots, j_{n}\right), \quad f_{i_{0}, i, j}=f_{i_{0}, \ldots, i_{n}, j_{1}, \ldots, j_{n}}, \\
f & =\sum_{i_{0}, i, j} f_{i_{0}, i, j} X_{0}^{i_{0}} X^{i} D^{j} .
\end{aligned}
$$


By definition,

$$
\begin{aligned}
& \operatorname{deg} f=\operatorname{deg}_{X_{0}, \ldots, X_{n}, D_{1}, \ldots, D_{n}} f=\max \left\{i_{0}+|i|+|j|: f_{i_{0}, i, j} \neq 0\right\}, \\
& \operatorname{deg}_{D_{1}, \ldots, D_{n}} f=\max \left\{|j|: f_{i_{0}, i, j} \neq 0\right\}, \\
& \operatorname{deg}_{D_{\alpha}} f=\max \left\{j_{\alpha}: f_{i_{0}, i, j} \neq 0\right\}, \quad 1 \leq \alpha \leq n \\
& \operatorname{deg}_{X_{\alpha}} f=\max \left\{i_{\alpha}: f_{i_{0}, i, j} \neq 0\right\}, \quad 1 \leq \alpha \leq n .
\end{aligned}
$$

Set ord $0=\operatorname{ord}_{X_{0}} 0=+\infty$. If $0 \neq f \in{ }^{h} A$, then we put

$$
\text { ord } f=\operatorname{ord}_{X_{0}} f=\mu \Longleftrightarrow f \in X_{0}^{\mu}\left({ }^{h} A\right) \backslash X_{0}^{\mu+1}\left({ }^{h} A\right), \quad \mu \geq 0 .
$$

For every $z=\left(z_{1}, \ldots, z_{l}\right) \in{ }^{h} A^{l}$, put

$$
\operatorname{ord} z=\min _{1 \leq i \leq l}\left\{\operatorname{ord} z_{i}\right\}, \quad \operatorname{deg} z=\max _{1 \leq i \leq l}\left\{\operatorname{deg} z_{i}\right\} .
$$

The quantities ord $b$ and $\operatorname{deg} b$ are defined similarly for an arbitrary $(k \times l)$-matrix $b$ with coefficients in ${ }^{h} A$. More precisely, here $b$ is viewed as a vector with $k l$ entries.

An element $f \in{ }^{h} A$ is homogeneous if and only if $f_{i_{0}, i, j} \neq 0$ implies $i_{0}+|i|+|j|=\operatorname{deg} f$, i.e., $f$ is a sum of monomials of the same degree $\operatorname{deg} f$. The homogeneous degree of a nonzero homogeneous element $f$ is its degree. The homogeneous degree of 0 is not defined (0 belongs to all the homogeneous components of ${ }^{h} A$; see below).

Next, for every integer $m$, the $m$ th homogeneous component of ${ }^{h} A$ is the $F$-linear space

$$
\left({ }^{h} A\right)_{m}=\left\{z \in{ }^{h} A: z \text { is homogeneous and } \operatorname{deg} z=m \text { or } z=0\right\} .
$$

Now ${ }^{h} A$ is a graded ring with respect to the homogeneous degree. By definition, the ring ${ }^{h} A$ is a homogenization of the Weyl algebra $A$.

We shall consider the category of finitely generated graded modules $G$ over the ring ${ }^{h} A$. Such a module $G=\bigoplus_{m \geq m_{0}} G_{m}$ is a direct sum of its homogeneous components $G_{m}$, where $m, m_{0}$ are integers. Every $G_{m}$ is a finite-dimensional $F$-linear space, and $\left({ }^{h} A\right)_{p} G_{m} \subset G_{p+m}$ for all integers $p, m$. Let $G$ and $G^{\prime}$ be two finitely generated graded ${ }^{h} A$-modules; then $\varphi: G \rightarrow G^{\prime}$ is a morphism (of degree 0) of graded modules if and only if $\varphi$ is a morphism of ${ }^{h} A$-modules and $\varphi\left(G_{m}\right) \subset G_{m}^{\prime}$ for every integer $m$.

An element $z \in{ }^{h} A$ (respectively, $z \in A$ ) is called a term if and only if $z=\lambda z_{1} \cdots z_{\nu}$ for some $0 \neq \lambda \in F$, some integer $\nu \geq 0$, and $z_{w} \in\left\{X_{0}, \ldots, X_{n}, D_{1}, \ldots, D_{n}\right\}$ (respectively, $\left.z_{w} \in\left\{X_{1}, \ldots, X_{n}, D_{1}, \ldots, D_{n}\right\}\right), 1 \leq w \leq \nu$.

Let $z=\sum_{j} z_{j} \in A$ be an arbitrary element of the Weyl algebra $A$ represented as a sum of terms $z_{j}$, and let $\operatorname{deg} z=\max _{j} \operatorname{deg} z_{j}$. For example, here we can take the representation (3) for $z$. Then we define the homogenization ${ }^{h} z \in{ }^{h} A$ by the formula

$$
h_{z}=\sum_{j} z_{j} X_{0}^{\operatorname{deg} z-\operatorname{deg} z_{j}} .
$$

By (2) and (13), the right-hand side of this relation does not depend on the choice of a representation of $z$ as a sum of terms. Hence, ${ }^{h} z$ is well defined. If $z \in{ }^{h} A$, then ${ }^{a} z \in A$ is obtained by substituting $X_{0}=1$ in $z$. Hence, for every $z \in A$ we have ${ }^{a h} z=z$, and for every $z \in{ }^{h} A$ we have $z={ }^{h a} z X_{0}^{\mu}$, where $\mu=\operatorname{ord} z$.

For an element $z=\left(z_{1}, \ldots, z_{l}\right) \in A^{l}$, we put $\operatorname{deg} z=\max _{1 \leq i \leq l}\left\{\operatorname{deg} z_{i}\right\}$ and

$$
h_{z}=\left({ }^{h} z_{1} X_{0}^{\operatorname{deg} z-\operatorname{deg} z_{1}}, \ldots,{ }_{z_{l}} X_{0}^{\operatorname{deg} z-\operatorname{deg} z_{l}}\right) \in{ }^{h} A^{l} .
$$

The degree $\operatorname{deg} a$ and the homogenization ${ }^{h} a$ can be defined similarly for an arbitrary $(k \times l)$-matrix $a=\left(a_{v, w}\right)_{1 \leq v \leq k, 1 \leq w \leq l}$ with coefficients in $A$. More precisely, here $a$ is viewed as a vector with $k l$ entries. Hence, if $b=\left(b_{v, w}\right)_{1 \leq v \leq k, 1 \leq w \leq l}={ }^{h} a$, then $b_{v, w}={ }^{h} a_{v, w} X_{0}^{\operatorname{deg} a-\operatorname{deg} a_{v, w}}$ for all $v, w$. 
Next, the $m$ th homogeneous component of ${ }^{h} A^{l}$ is

$$
\left({ }^{h} A^{l}\right)_{m}=\left\{{ }^{h} z: z \in A^{l} \text { and } \operatorname{deg} z=m \text { or } z=0\right\} .
$$

For an $F$-linear subspace $X \subset A^{l}$, we let ${ }^{h} X$ be the smallest linear subspace of ${ }^{h} A^{l}$ containing the set $\left\{{ }^{h} z: z \in X\right\}$. If $X$ is an $A$-submodule of $A^{l}$, then ${ }^{h} X$ is a graded submodule of ${ }^{h} A^{l}$. The graduation on ${ }^{h} X$ is induced by that of ${ }^{h} A^{l}$.

For an element $z=\left(z_{1}, \ldots, z_{l}\right) \in{ }^{h} A^{l}$, put ${ }^{a} z=\left({ }^{a} z_{1}, \ldots,{ }^{a} z_{l}\right) \in A^{l}$. For a subset $X \subset{ }^{h} A^{l}$, put ${ }^{a} X=\left\{{ }^{a} z: z \in X\right\} \subset A^{l}$. If $X$ is an $F$-linear space, then ${ }^{a} X$ is also an $F$-linear space. If $X$ is a graded submodule of ${ }^{h} A^{l}$, then ${ }^{a} X$ is a submodule of $A^{l}$.

Now, ${ }^{h} I$ is a graded submodule of ${ }^{h} A^{l}$, and ${ }^{a h} I=I$. Let $\left({ }^{h} I\right)_{m}$ be the $m$ th homogeneous component of ${ }^{h} I$. Then

$$
\begin{aligned}
{ }^{h}\left(I_{m}\right) & =\bigoplus_{0 \leq j \leq m}\left({ }^{h} I\right)_{j}, \quad m \geq 0, \\
{ }^{a}\left(\left({ }^{h} I\right)_{m}\right) & =I_{m}, \quad m \geq 0,
\end{aligned}
$$

and (18) induces an isomorphism $\iota:\left({ }^{h} I\right)_{m} \rightarrow I_{m}$ of linear spaces over $F$. Set ${ }^{h} M=$ ${ }^{h} A^{l} /{ }^{h} I$. Then ${ }^{h} M$ is a graded ${ }^{h} A$-module, and we have the exact sequence

$$
0 \rightarrow{ }^{h} I \rightarrow{ }^{h} A^{l} \rightarrow{ }^{h} M \rightarrow 0 .
$$

Now, for the $m$ th homogeneous component $\left({ }^{h} M\right)_{m}$ of ${ }^{h} M$ we have

$$
\left({ }^{h} M\right)_{m}=\left({ }^{h} A^{l}\right)_{m} /\left({ }^{h} I\right)_{m} \simeq A_{m}^{l} / I_{m},
$$

by the isomorphism $\iota$. We have the exact sequences

$$
0 \rightarrow\left({ }^{h} I\right)_{m} \rightarrow\left({ }^{h} A^{l}\right)_{m} \rightarrow\left({ }^{h} M\right)_{m} \rightarrow 0, \quad m \geq 0 .
$$

By definition, the Hilbert function of the module ${ }^{h} M$ is

$$
H\left({ }^{h} M, m\right)=\operatorname{dim}_{F}\left({ }^{h} M\right)_{m}, \quad m \geq 0 .
$$

By (20), we have $H(M, m)=H\left({ }^{h} M, m\right)$ for every $m \geq 0$; i.e., the Hilbert functions of $M$ and ${ }^{h} M$ coincide.

Lemma 2. Let $b_{1}, \ldots, b_{s}$ be a system of homogeneous generators of the ${ }^{h} A$-module ${ }^{h} I$. Then

$$
\operatorname{gr}\left({ }^{a} b_{1}\right), \ldots, \operatorname{gr}\left({ }^{a} b_{s}\right) \in \operatorname{gr}(A)^{l}
$$

is a system of generators of the $\operatorname{gr}(A)$-module $\operatorname{gr}(I)$.

Proof. By (18), we have ${ }^{a}\left(\left({ }^{h} I\right)_{m}\right)=I_{m}$. Now the claim follows from Lemma 1 . The lemma is proved.

\section{$\S 4$. The Janet Bases of A MOdule AND OF ITS HOMOGENIZATION}

Each element of ${ }^{h} A^{l}$ can be uniquely represented as an $F$-linear combination of elements $e_{v, i_{0}, i, j}=\left(0, \ldots, 0, X_{0}^{i_{0}} X^{i} D^{j}, 0, \ldots, 0\right)$, where $0 \leq i_{0} \in \mathbb{Z}, i, j \in \mathbb{Z}_{+}^{n}$ are multiindices, see (4), and the nonzero monomial $X_{0}^{i_{0}} X^{i} D^{j}$ is at the position $v, 1 \leq v \leq l$. Therefore, every element $f \in{ }^{h} A^{l}$ can be written in the form

$$
f=\sum_{v, i_{0}, i, j} f_{v, i_{0}, i, j} e_{v, i_{0}, i, j}, \quad f_{v, i_{0}, i, j} \in F,
$$

and only a finite number of $f_{v, i_{0}, i, j}$ are nonzero. The elements $e_{v, i_{0}, i, j}$ will be called monomials.

In $\S 1$, everywhere after the definition of the Hilbert function, we can replace the ring $A$, the monomials $e_{v, i, j}$, the multi-indices $i, i^{\prime}, i^{\prime \prime}$, the triples $(v, i, j)$ and $\left(v, i^{\prime}, j^{\prime}\right)$, the module $I$, and so on by the ring ${ }^{h} A$, the monomials $e_{v, i_{0}, i, j}$, the pairs $\left(i_{0}, i\right),\left(i_{0}^{\prime}, i^{\prime}\right)$, 
$\left(i_{0}^{\prime \prime}, i^{\prime \prime}\right)$ (they are used without parentheses), the quadruples $\left(v, i_{0}, i, j\right),\left(v, i_{0}^{\prime}, i^{\prime}, j^{\prime}\right)$, the homogenization ${ }^{h} I$, and so on, respectively. This gives us the definitions of $o(f), \operatorname{Hdt}(f)$ for $f \in{ }^{h} A^{l}$, new conditions (a) and (b) that define admissible linear orders on the monomials of ${ }^{h} A^{l}$, a new condition (c) and the definition of the degree-compatible linear order, new conditions 1)-5), and the definitions of the set $\operatorname{Hdt}\left({ }^{h} I\right)$, the Janet basis, and the reduced Janet basis of ${ }^{h} I$. For example, $o(0)=+\infty$, $\operatorname{Hdt}(0)=0$, and if $0 \neq f \in{ }^{h} A^{l}$, then

$$
\begin{aligned}
o(f) & =\max \left\{\left(v, i_{0}, i, j\right): f_{v, i_{0}, i, j} \neq 0\right\}, \\
\operatorname{Hdt}(f) & =f_{v, i_{0}, i, j} e_{v, i_{0}, i, j}, \quad \text { where } o(f)=\left(v, i_{0}, i, j\right), \\
\operatorname{Hdt}\left({ }^{h} I\right) & =\left\{\operatorname{Hdt}(f): f \in{ }^{h} I\right\},
\end{aligned}
$$

while the new conditions (a) and (b) look like this:

(a) for all indices $i_{0}, i_{0}^{\prime}$, all multi-indices $i, j, i^{\prime}, j^{\prime}$, and all $1 \leq v \leq l$, if $i_{0} \leq i_{0}^{\prime}$, $i_{1} \leq i_{1}^{\prime}, \ldots, i_{n} \leq i_{n}^{\prime}$, and $j_{1} \leq j_{1}^{\prime}, \ldots, j_{n} \leq j_{n}^{\prime}$, then $\left(v, i_{0}, i, j\right) \leq\left(v, i_{0}^{\prime}, i^{\prime}, j^{\prime}\right)$

(b) for all indices $i_{0}, i_{0}^{\prime}, i_{0}^{\prime \prime}$, all multi-indices $i, j, i^{\prime}, j^{\prime}, i^{\prime \prime}, j^{\prime \prime}$, and all $1 \leq v, v^{\prime} \leq l$, if $\left(v, i_{0}, i, j\right)<\left(v^{\prime}, i_{0}^{\prime}, i^{\prime}, j^{\prime}\right)$, then $\left(v, i_{0}+i_{0}^{\prime \prime}, i+i^{\prime \prime}, j+j^{\prime \prime}\right)<\left(v^{\prime}, i_{0}^{\prime}+i_{0}^{\prime \prime}, i^{\prime}+i^{\prime \prime}, j^{\prime}+j^{\prime \prime}\right)$.

The existence of a Janet basis of ${ }^{h} I$ and the uniqueness of the reduced Janet basis with respect to an admissible linear order are proved much as the existence of a Janet basis of $I$ and the uniqueness of the reduced Janet basis of $I$; see $\S 1$. The Janet basis of ${ }^{h} I$ is homogeneous if and only if it consists of homogeneous elements of ${ }^{h} A^{l}$. Since the module ${ }^{h} I$ is homogeneous, the family of homogeneous components of any Janet basis of ${ }^{h} I$ is a homogeneous Janet basis of ${ }^{h} I$. Hence, the reduced Janet basis of ${ }^{h} I$ is homogeneous (here we leave the details to the reader).

Let $<$ be an admissible linear order on the monomials in $A^{l}$, or, what is the same, on the triples $(v, i, j)$; see $\S 1$. Thus, this order satisfies conditions (a) and (b). We define a linear order on the monomials $e_{v, i_{0}, i, j}$, or, what is the same, on the quadruples $\left(v, i_{0}, i, j\right)$. This linear order is induced by $<$ on the triples $(v, i, j)$ and will be denoted again by $<$. Namely, for two quadruples $\left(v, i_{0}, i, j\right)$ and $\left(v^{\prime}, i_{0}^{\prime}, i^{\prime}, j^{\prime}\right)$ we put $\left(v, i_{0}, i, j\right)<\left(v^{\prime}, i_{0}^{\prime}, i^{\prime}, j^{\prime}\right)$ if and only if $(v, i, j)<\left(v^{\prime}, i^{\prime}, j^{\prime}\right)$, or $(v, i, j)=\left(v^{\prime}, i^{\prime}, j^{\prime}\right)$ but $i_{0}<i_{0}^{\prime}$. Observe that this induced linear order satisfies conditions (a) and (b) (in the new sense).

Remark 2. If $f_{1}, \ldots, f_{m}$ is a Janet basis of $I$ (respectively, a homogeneous Janet basis of ${ }^{h} I$ ) satisfying 1)-4), then there are unique $c_{\alpha, \beta} \in A$ (respectively, homogeneous $c_{\alpha, \beta} \in$ $\left.{ }^{h} A\right), 1 \leq \alpha<\beta \leq m$, such that the elements

$$
f_{\alpha}+\sum_{\alpha<\beta \leq m} c_{\alpha, \beta} f_{\beta}, \quad 1 \leq \alpha \leq m,
$$

form a reduced Janet basis of $I$ (respectively, a reduced homogeneous Janet basis of ${ }^{h} I$ ); cf. 3].

Obviously, an admissible linear order $<$ on the monomials in $A^{l}$ (respectively, in ${ }^{h} A^{l}$ ) is degree-compatible if and only if for any two monomials $z_{1}, z_{2}$ the inequality $\operatorname{deg} z_{1}<\operatorname{deg} z_{2}$ implies $z_{1}<z_{2}$.

Lemma 3. The following assertions are true.

(i) Let $f_{1}, \ldots, f_{m}$ be a (reduced) Janet basis of I with respect to the linear order $<$ and suppose that the order $<$ is degree-compatible. Then ${ }^{h} f_{1}, \ldots,{ }^{h} f_{m}$ is a (reduced) homogeneous Janet basis of the module ${ }^{h} I$ with respect to the induced linear order $<$. 
(ii) Conversely, suppose that the initial order $<$ is degree-compatible, and $g_{1}, \ldots, g_{m}$ is a (reduced) homogeneous Janet basis of the module ${ }^{h} I$ with respect to the induced linear order $<$. Then ${ }^{a} g_{1}, \ldots,{ }^{a} g_{m}$ is a (reduced) Janet basis of $I$ with respect to the linear order $<$.

(iii) Suppose that the initial order $<$ is arbitrary admissible. Let $g_{1}, \ldots, g_{m}$ be a homogeneous Janet basis of the module ${ }^{h} I$ with respect to the induced linear order $<$. Then ${ }^{a} g_{1}, \ldots,{ }^{a} g_{m}$ is a Janet basis of $I$ with respect to the linear order $<$. Moreover, ${ }^{h a} g_{w}=g_{w}$ for all $1 \leq w \leq m$.

Proof. This follows immediately from the definitions.

Let $f \in{ }^{h} A^{l}$, and let the module ${ }^{h} I$ be as above. We show that there is a unique element $g \in{ }^{h} A^{l}$ such that

$$
g=\sum_{v, i_{0}, i, j} g_{v, i_{0}, i, j} e_{v, i_{0}, i, j}, \quad g_{v, i_{0}, i, j} \in F
$$

$f-g \in{ }^{h} I$, and if $g_{v, i_{0}, i, j} \neq 0$, then $e_{v, i_{0}, i, j} \notin \operatorname{Hdt}\left({ }^{h} I\right)$. Indeed, if there are two such elements $g \neq g^{\prime}$, then $0 \neq g-g^{\prime} \in{ }^{h} I$, but $\operatorname{Hdt}\left(g-g^{\prime}\right) \notin \operatorname{Hdt}\left({ }^{h} I\right)$, and we get a contradiction. To prove the existence of $g$, we may assume without loss of generality that $f$ is homogeneous and show additionally that the sum on the left in (23) is taken over $\left(v, i_{0}, i, j\right)$ such that $i_{0}+|i|+|j|=\operatorname{deg} f$. We can write

$$
f=\sum_{v, i_{0}, i, j} f_{v, i_{0}, i, j} e_{v, i_{0}, i, j}, \quad f_{v, i_{0}, i, j} \in F, \quad i_{0}+|i|+|j|=\operatorname{deg} f .
$$

We use induction on the number $\nu(f)$ of quadruples $\left(v, i_{0}, i, j\right)$ in the last sum such that $e_{v, i_{0}, i, j} \in \operatorname{Hdt}\left({ }^{h} I\right)$ and $e_{v, i_{0}, i, j} \leq \operatorname{Hdt}(f)$. If $\nu(f)>0$, then there is a homogeneous $z \in{ }^{h} I$ such that $\operatorname{Hdt}(z)=\operatorname{Hdt}(f), \operatorname{deg} z=\operatorname{deg} f$. Then $\nu(f-z)<\nu(f)$. The required assertion is proved.

The element $g$ as in (23) is called the normal form of $f$ with respect to the module ${ }^{h} I$. We denote $g=\operatorname{nf}\left({ }^{h} I, f\right)$. Obviously, $\operatorname{nf}\left({ }^{h} I,\left({ }^{h} A^{l}\right)_{m}\right) \subset\left({ }^{h} A^{l}\right)_{m}$ is a linear subspace, and

$$
\operatorname{dim}_{F} \operatorname{nf}\left({ }^{h} I,\left({ }^{h} A^{l}\right)_{m}\right)=l\left(\begin{array}{c}
m+2 n \\
2 n
\end{array}\right)-H\left({ }^{h} I, m\right)=H\left({ }^{h} A^{l} /{ }^{h} I, m\right) .
$$

Let ${ }^{c} A=F\left[X_{0}, \ldots, X_{n}, D_{1}, \ldots, D_{n}\right]$ denote the polynomial ring in the variables $X_{0}, \ldots, X_{n}, D_{1}, \ldots, D_{n}$. Each monomial $e_{v, i_{0}, i, j}$ can also be viewed as an element of ${ }^{c} A^{l}$. Hence, $\operatorname{Hdt}(f)$ can be viewed as an element of ${ }^{c} A^{l}$ for every $f \in{ }^{h} A^{l}$. To avoid ambiguity, we shall denote it by $\operatorname{Hdtc}(f) \in{ }^{c} A^{l}$. $\operatorname{Put} \operatorname{Hdtc}\left({ }^{h} I\right)=\left\{\operatorname{Hdtc}(f): f \in{ }^{h} I\right\}$. So, the sets $\operatorname{Hdt}\left({ }^{h} I\right)$ and $\operatorname{Hdtc}\left({ }^{h} I\right)$ are in one-to-one correspondence.

We denote by ${ }^{c} I \subset{ }^{c} A^{l}$ the graded submodule of ${ }^{c} A^{l}$ generated by $\operatorname{Hdtc}\left({ }^{h} I\right)$. It is easily seen that the set of monomials from the module ${ }^{c} I$ coincides with $\operatorname{Hdtc}\left({ }^{h} I\right) \backslash\{0\}$. Next, for every $m \geq 0$, the $F$-linear space ${ }^{c} I_{m}$ of homogeneous elements is generated by the monomials $e_{v, i_{0}, i, j}$ such that there is $0 \neq f \in{ }^{h} I_{m}$ with $o(f)=\left(v, i_{0}, i, j\right)$. For the Hilbert function, we have

$$
\begin{aligned}
H\left({ }^{c} I, m\right) & =\operatorname{dim}_{F}\left\{\left(z_{1}, \ldots, z_{l}\right) \in{ }^{c} I: \forall i\left(\operatorname{deg} z_{i}=m \text { or } z_{i}=0\right)\right\}, \\
H\left({ }^{c} A^{l} /{ }^{c} I, m\right) & =l\left(\begin{array}{c}
m+2 n \\
2 n
\end{array}\right)-H\left({ }^{c} I, m\right) .
\end{aligned}
$$

Let $f \in{ }^{c} A^{l}$, and let the module ${ }^{c} I$ be as above. Then there is a unique element $g \in{ }^{c} A^{l}$ such that

$$
g=\sum_{v, i_{0}, i, j} g_{v, i_{0}, i, j} e_{v, i_{0}, i, j}, \quad g_{v, i_{0}, i, j} \in F
$$


$f-g \in{ }^{h} I$, and if $g_{v, i_{0}, i, j} \neq 0$, then $e_{v, i_{0}, i, j} \notin \operatorname{Hdtc}\left({ }^{h} I\right)$ (the proof is similar to that of the existence and uniqueness of $g$ in (23)). The element $g$ is called the normal form of $f$ with respect to the module ${ }^{c} I$; see [4. We denote $g=\operatorname{nf}\left({ }^{c} I, f\right)$. Obviously, $\operatorname{nf}\left({ }^{c} I,\left({ }^{c} A^{l}\right)_{m}\right) \subset$ $\left({ }^{c} A^{l}\right)_{m}$ is a linear subspace, and

$$
\operatorname{dim}_{F} \operatorname{nf}\left({ }^{c} I,\left({ }^{c} A^{l}\right)_{m}\right)=l\left(\begin{array}{c}
m+2 n \\
2 n
\end{array}\right)-H\left({ }^{c} I, m\right)=H\left({ }^{c} A^{l} /{ }^{c} I, m\right) .
$$

Since, by the definitions given above, the $F$-linear spaces $\operatorname{nf}\left({ }^{c} I,\left({ }^{c} A^{l}\right)_{m}\right)$ and $\operatorname{nf}\left({ }^{h} I,\left({ }^{h} A^{l}\right)_{m}\right)$ are generated by the same monomials, for every $m \geq 0$ we have

$$
\begin{aligned}
\operatorname{dim}_{F} \operatorname{nf}\left({ }^{c} I,\left({ }^{c} A^{l}\right)_{m}\right) & =\operatorname{dim}_{F} \operatorname{nf}\left({ }^{h} I,\left({ }^{h} A^{l}\right)_{m}\right), \\
H\left({ }^{h} A^{l} /{ }^{h} I, m\right) & =H\left({ }^{c} A^{l} /{ }^{c} I, m\right), \quad H\left({ }^{h} I, m\right)=H\left({ }^{c} I, m\right) .
\end{aligned}
$$

Therefore, see $\S 3$,

$$
H(I, m)=H\left({ }^{c} I, m\right), \quad m \geq 0 .
$$

§5. Bound FOR THE KERNEL OF A MATRIX OVER THE HOMOGENIZED WEYL ALGEBRA

Lemma 4. Let $k \geq 1$ and $l \geq 1$ be integers. Let $b=\left(b_{i, j}\right)_{1 \leq i \leq k, 1 \leq j \leq l}$ be a matrix, where $b_{i, j} \in{ }^{h} A$ are homogeneous elements for all $i, j$. Suppose $\operatorname{deg} b_{i, j}<d, d \geq 2$, for all $i, j$. Assume that there are integers $d_{j} \geq 0,1 \leq i \leq k$, and $d_{i}^{\prime} \geq 0,1 \leq j \leq l$, such that

$$
\operatorname{deg} b_{i, j}=d_{i}-d_{j}^{\prime}
$$

for all nonzero $b_{i, j}$, and that, moreover, the $d_{j}^{\prime}$ are chosen to be minimal possible (this means that there are no integers $\widetilde{d}_{i}, \widetilde{d}_{j}^{\prime}$ similar to $d_{i}, d_{j}^{\prime}$ such that $\widetilde{d}_{j}^{\prime} \leq d_{j}^{\prime}$ for all $1 \leq$ $j \leq l$ and at least one of the inequalities is strict). Then $d_{i}<\min \{k+1, l\} d$ and $d_{j}^{\prime}<\min \{k, l-1\} d$ for all $i, j$.

Next, assume that $k=l-1$. Then there are homogeneous elements $z_{1}, \ldots, z_{l} \in{ }^{h} A$ such that $\left(z_{1}, \ldots, z_{l}\right) \neq(0, \ldots, 0)$ and

$$
\sum_{1 \leq j \leq l} b_{i, j} z_{j}=0, \quad 1 \leq i \leq l-1 .
$$

There is an integer $\mu \geq 0$ such that, for all $1 \leq j \leq l-1$, if $z_{j} \neq 0$, then $\operatorname{deg} z_{j}=$ $\mu+d_{j}^{\prime}$, and hence, all nonzero $b_{i, j} z_{j}$ have one and the same degree depending only on $i$. Furthermore,

$$
\operatorname{deg} z_{j} \leq(2 n+1) l \max _{1 \leq i \leq k}\left\{d_{i}\right\}<(2 n+1) l^{2} d, \quad 1 \leq j \leq l .
$$

Moreover, if all $b_{i, j}$ do not depend on $X_{n}$ (i.e., they can be represented as sums of monomials that do not contain $X_{n}$ ), then the elements $z_{1}, \ldots, z_{l}$ can be chosen so as to satisfy additionally the same property. Finally, dividing by an appropriate power of $X_{0}$, we can assume without loss of generality that $\min \left\{\operatorname{ord} z_{i}: 1 \leq i \leq l\right\}=0$.

Proof. First, we prove that $d_{i}<\min \{k+1, l\} d$ and $d_{j}^{\prime}<\min \{k, l-1\} d$ for all $i, j$ and arbitrary $k, l \geq 1$. We define an equivalence relation on the set of pairs $P=\{(v, w)$ : $1 \leq v \leq k, 1 \leq w \leq l$, and $\left.b_{v, w} \neq 0\right\}$ as follows. Put $(v, w) \sim\left(v^{\prime}, w^{\prime}\right)$ if and only if in $P$ there is a sequence of pairs $\left(v_{1}, w_{1}\right), \ldots,\left(v_{\nu}, w_{\nu}\right), \nu \geq 1$, such that

1) $(v, w)=\left(v_{1}, w_{1}\right),\left(v^{\prime}, w^{\prime}\right)=\left(v_{\nu}, w_{\nu}\right)$,

2) $v_{\alpha}=v_{\alpha+1}$ or $w_{\alpha}=w_{\alpha+1}$ for every $1 \leq \alpha \leq \nu-1$.

Let $\pi \subset P$ be an equivalence class with respect to $\sim$. Then there is a pair $(p, q) \in \pi$ such that $d_{q}^{\prime}=0$, because the numbers $d_{j}^{\prime}$ are chosen to be minimal possible. Moreover, for all $(v, w),\left(v^{\prime}, w^{\prime}\right) \in \pi$, a sequence $\left(v_{1}, w_{1}\right), \ldots,\left(v_{\nu}, w_{\nu}\right)$ as above can always be chosen so 
as to possess the following five properties:

3) $\left(v_{\alpha}, w_{\alpha}\right) \neq\left(v_{\alpha+1}, w_{\alpha+1}\right)$ for every $1 \leq \alpha \leq \nu-1$;

4) if $v_{\alpha}=v_{\alpha+1}$, then $w_{\alpha+1}=w_{\alpha+2}$ for every $1 \leq \alpha \leq \nu-2$;

5) if $w_{\alpha}=w_{\alpha+1}$, then $v_{\alpha+1}=v_{\alpha+2}$ for every $1 \leq \alpha \leq \nu-2$;

6) for all $1 \leq \alpha, \beta \leq \nu$, if $\beta \notin\{\alpha-1, \alpha, \alpha+1\}$, then $v_{\beta} \neq v_{\alpha}$;

7) for all $1 \leq \alpha, \beta \leq \nu$, if $\beta \notin\{\alpha-1, \alpha, \alpha+1\}$, then $w_{\beta} \neq w_{\alpha}$

(we leave the details to the reader). Now conditions 1)-7) imply that the number of pairs satisfies

$$
\#\left\{\left(w_{\alpha}, w_{\alpha+1}\right): w_{\alpha} \neq w_{\alpha+1} \& 1 \leq \alpha \leq \nu-1\right\} \leq \min \{k, l-1\} .
$$

Next, if $w_{\alpha} \neq w_{\alpha+1}$, then $v_{\alpha+1}=v_{\alpha}$ and $\left|d_{w_{\alpha+1}}^{\prime}-d_{w_{\alpha}}^{\prime}\right|=\left|\operatorname{deg} b_{v_{\alpha+1}, w_{\alpha+1}}-\operatorname{deg} b_{v_{\alpha}, w_{\alpha}}\right|<$ $d$. Hence, $d_{w_{\nu}}^{\prime}<\min \{k, l-1\} d+d_{w_{1}}^{\prime}$. For $\left(v_{1}, w_{1}\right)=(p, q)$ and an arbitrary $(v, w)=$ $\left(v_{\nu}, w_{\nu}\right) \in \pi$ we get $d_{w}^{\prime}<\min \{k, l-1\} d$. Finally, $\operatorname{deg} b_{v, w}=d_{v}-d_{w}^{\prime}<d$ implies $d_{v}<\min \{k+1, l\} d$. The required inequalities are proved.

Now, suppose that $\operatorname{deg} b_{i, j}=\operatorname{deg} b$ for all nonzero $b_{i, j}$ and $k=l-1$. We prove the existence of $z_{1}, \ldots, z_{l}$ and obtain an estimate for $\operatorname{deg} z_{j}$ in this case. Consider the linear mapping

$$
\begin{aligned}
\left({ }^{h} A\right)_{m-\operatorname{deg} b}^{l} & \longrightarrow\left({ }^{h} A\right)_{m}^{l-1}, \\
\left(z_{1}, \ldots, z_{l}\right) & \mapsto\left(\sum_{1 \leq j \leq l} b_{i, j} z_{j}\right)_{1 \leq i \leq l-1} .
\end{aligned}
$$

If

$$
l\left(\begin{array}{c}
m-\operatorname{deg} b+2 n \\
2 n
\end{array}\right)>(l-1)\left(\begin{array}{c}
m+2 n \\
2 n
\end{array}\right),
$$

then the kernel of (28) is nonzero. But (29) is true provided

$$
\prod_{1 \leq w \leq 2 n}\left(1+\frac{\operatorname{deg} b}{m+w-\operatorname{deg} b}\right)<\frac{l}{l-1} .
$$

Next, (30) is true if $(1+\operatorname{deg} b /(m-\operatorname{deg} b))^{2 n}<l /(l-1)$. The last inequality follows from $m \geq(2 n+1) \operatorname{deg} b / \log (l /(l-1))$, and hence, also from $m \geq(2 n+1) l \operatorname{deg} b$. Thus, the existence of $z_{1}, \ldots, z_{l}$ is proved, and moreover, all the nonzero $z_{j}$ have one and the same degree $((2 n+1) l-1) \operatorname{deg} b$, which does not depend on $j$. Observe that, in the case under consideration we have proved a stronger inequality: $\operatorname{deg} z_{j}<((2 n+1) l-1) d$ for all $1 \leq j \leq l$.

Finally, let $k=l-1$ and suppose that the degrees $\operatorname{deg} b_{i, j}$ are arbitrary but satisfy (25). Multiplying the $i$ th equation in (26) by $X_{0}^{\max _{w}\left\{d_{w}\right\}-d_{i}}$, we may assume without loss of generality that all $d_{i}$ are equal. We substitute $z_{j} X_{0}^{d_{j}^{\prime}}$ for $z_{j}$ in (26). Now the degrees of all the nonzero coefficients of the resulting system are equal to $\max _{1 \leq i \leq k}\left\{d_{i}\right\}$ and are less than $l d$. If, in the case of $\operatorname{deg} b_{i, j}=\operatorname{deg} b$ considered above, we replace the bound $d$ by $\max _{1 \leq i \leq k}\left\{d_{i}\right\}<l d$, we get the required $z_{1}, \ldots, z_{l}$ such that $\operatorname{deg} z_{j}=$ $((2 n+1) l-1) \max _{1 \leq i \leq k}\left\{d_{i}\right\}+d_{j}^{\prime}$ or $z_{j}=0$ for all $1 \leq j \leq l$, together with the estimate

$$
\operatorname{deg} z_{j} \leq((2 n+1) l-1) \max _{1 \leq i \leq k}\left\{d_{i}\right\}+d_{j}^{\prime} \leq(2 n+1) l \max _{1 \leq i \leq k}\left\{d_{i}\right\}<(2 n+1) l^{2} d
$$

for all $j$.

Suppose that $a_{1}, \ldots, a_{l}$ do not depend on $X_{n}$. We represent $z_{i}=\sum_{j} z_{i, j} X_{n}^{j}, 1 \leq i \leq l$, where all $z_{i, j}$ do not depend on $X_{n}$. Let $\alpha=\max _{i}\left\{\operatorname{deg}_{X_{n}} z_{i}\right\}$. Obviously, in this case we can replace $\left(z_{1}, \ldots, z_{l}\right)$ by $\left(z_{1, \alpha}, \ldots, z_{l, \alpha}\right)$. The lemma is proved. 
Remark 3. Lemma 4 remains true if condition (26) in its statement is replaced by

$$
\sum_{1 \leq j \leq l} z_{j} b_{i, j}=0, \quad 1 \leq i \leq l-1 .
$$

The proof is similar.

Remark 4. Let the elements $b_{i, j}$ be as in Lemma 4 . Notice that there are integers $\delta_{i}^{\prime} \geq 0$, $1 \leq i \leq k$, and $\delta_{j} \geq 0,1 \leq j \leq l$, such that

$$
\operatorname{deg} b_{i, j}=\delta_{j}-\delta_{i}^{\prime}
$$

for all nonzero $b_{i, j}$, and $\min _{1 \leq i \leq k}\left\{\delta_{i}^{\prime}\right\}=0$. Namely, $\delta_{i}^{\prime}=-d_{i}+\max _{1 \leq i \leq k}\left\{d_{i}\right\}, \delta_{j}=$ $-d_{j}^{\prime}+\max _{1 \leq i \leq k}\left\{d_{i}\right\}$.

Remark 5. Let $b_{i, j} \in{ }^{h} A, 1 \leq i \leq k, 1 \leq j \leq l$, be homogeneous elements. Suppose there are integers $\widetilde{d}_{i}, 1 \leq i \leq k$, and $\widetilde{d}_{j}^{\prime}, 1 \leq j \leq l$, such that $\operatorname{deg} b_{i, j}=\widetilde{d}_{i}-\widetilde{d}_{j}^{\prime}$ for all nonzero $b_{i, j}$. Then there are integers $d_{i} \geq 0,1 \leq i \leq k$, and $d_{j}^{\prime} \geq 0,1 \leq j \leq l$, such that (25) is fulfilled for all nonzero $b_{i, j}$.

\section{§6. Reducing a matrix With COEFFicients in ${ }^{h} A$ to a TRAPEZOidal Form}

Let $b$ be a matrix as in Lemma 4 , and let integers $k, l \geq 1$ be arbitrary. Thus, (25) is true. Let $b=\left(b_{1}, \ldots, b_{l}\right)$, where $b_{1}, \ldots, b_{l} \in{ }^{h} A^{k}$, be the columns of the matrix $b$ (note that in Lemmas 1 and 2 all $b_{i}$ are rows of size $l$, so that now we change the notation). By definition, $b_{1}, \ldots, b_{l}$ are linearly independent over ${ }^{h} A$ from the right (or linearly independent if this will not lead to ambiguity; in what follows in this paper, if it is not stated otherwise, "linearly independent" will mean "linearly independent from the right") if and only if for all $z_{1}, \ldots, z_{l} \in{ }^{h} A$ the relation $b_{1} z_{1}+\cdots+b_{l} z_{l}=0$ implies $z_{1}=\cdots=z_{l}=0$. By (25), in this definition we may consider only homogeneous $z_{1}, \ldots, z_{l}$. From an arbitrary family $b_{1}, \ldots, b_{l}$ as in Lemma 4 (with arbitrary $k, l$ ) we can choose a maximal subfamily $b_{i_{1}}, \ldots, b_{i_{r}}$, linearly independent from the right. By Lemma 4, we have $r \leq k$. It turns out that $r$ does not depend on the choice of a subfamily. More precisely, the following statement is true.

Lemma 5. Let $c_{j}=\sum_{1 \leq i \leq l} b_{i} z_{i, j}, 1 \leq j \leq r_{1}$, where $z_{i, j} \in{ }^{h} A$ are homogeneous elements. Suppose that there are integers $d_{j}^{\prime \prime}, 1 \leq j \leq r_{1}$, such that, for all $i, j, \operatorname{deg} z_{i, j}=$ $d_{i}^{\prime}-d_{j}^{\prime \prime}$ if $z_{i, j} \neq 0$. Assume that $c_{j}, 1 \leq j \leq r_{1}$, are linearly independent over ${ }^{h} A$ from the right. Then $r_{1} \leq r$, and if $r_{1}<r$, then there are $c_{r_{1}+1}, \ldots, c_{r} \in\left\{b_{i_{1}}, \ldots, b_{i_{r}}\right\}$ such that the $c_{j}, 1 \leq j \leq r$, are linearly independent over ${ }^{h} A$ from the right.

Proof. The proof is similar to the case of vector spaces over a field, and we leave it to the reader.

We denote $r=\operatorname{rankr}\left\{b_{1}, \ldots, b_{l}\right\}$ and call this number the rank from the right of $b_{1}, \ldots, b_{l}$. In a similar way we can define the rank from the left of $b_{1}, \ldots, b_{l}$, denoting it by $\operatorname{rankl}\left\{b_{1}, \ldots, b_{l}\right\}$. It is not difficult to construct examples when $\operatorname{rankr}\left\{b_{1}, \ldots, b_{l}\right\}$ $\neq \operatorname{rankl}\left\{b_{1}, \ldots, b_{l}\right\}$. Our aim in this section is to prove the following result.

Lemma 6. Let $b$ be a matrix with homogeneous entries in ${ }^{h} A$ and satisfying (25); see above. Suppose that $d \geq 2$ and $\operatorname{deg} b_{i, j}<d$ for all $i, j$. Let $l_{1}=\operatorname{rankr}\left\{b_{1}, \ldots, b_{l}\right\}$, and let $b_{1}, \ldots, b_{l_{1}}$ be linearly independent. Hence, $0 \leq l_{1} \leq l$ and $k \geq l_{1}$. Then there is a matrix $\left(z_{j, r}\right)_{1 \leq j, r \leq l_{1}}$ (if $l_{1}=0$, then this matrix is empty) with homogeneous entries $z_{j, r} \in{ }^{h} A$, 
and a square permutation matrix $\sigma$ of size $k$, with the following properties:

(i) There are integers $d_{r}^{\prime \prime}, 1 \leq r \leq l_{1}$, such that for all $1 \leq j, r \leq l_{1}$ we have $\operatorname{deg} z_{j, r}=d_{j}^{\prime}-d_{r}^{\prime \prime}$ or $z_{j, r}=0$, and hence, all the nonzero elements $b_{i, j} z_{j, r}$, $1 \leq j \leq l_{1}$, have one and the same degree $d_{i}-d_{r}^{\prime \prime}$ depending only on $i, r$. Next,

$$
\operatorname{deg} z_{j, r} \leq(2 n+1) l_{1} \max _{1 \leq i \leq k}\left\{d_{i}\right\}<(2 n+1) l_{1}^{2} d .
$$

(ii) Introduce the matrix $e=\left(e_{i, j}\right)_{1 \leq i \leq k, 1 \leq j \leq l_{1}}=\sigma\left(b_{1}, \ldots, b_{l_{1}}\right) z$, where $\left(b_{1}, \ldots, b_{l_{1}}\right)$ is the matrix formed by the first $l_{1}$ columns of the matrix $b$. Then

$$
e=\left(\begin{array}{c}
e^{\prime} \\
e^{\prime \prime}
\end{array}\right) \text {, }
$$

where $e^{\prime}=\operatorname{diag}\left(e_{1,1}^{\prime}, \ldots, e_{l_{1}, l_{1}}^{\prime}\right)$ is a diagonal matrix with $l_{1}$ columns, and each $e_{j, j}^{\prime}, 1 \leq j \leq l_{1}$, is nonzero.

(iii) ord $e_{i, j} \geq$ ord $e_{j, j}^{\prime}$ for all $1 \leq i \leq k, 1 \leq j \leq l_{1}$.

Moreover, if all $a_{i, j}$ (and hence, all $b_{i, j}$ ) do not depend on $X_{n}$ (i.e., they can be represented as sums of monomials that do not contain $X_{n}$ ), then the $z_{j, r}$ can be chosen so as to possess the same property. Finally, dividing by an appropriate power of $X_{0}$, we may assume without loss of generality that $\min \left\{\operatorname{ord} z_{j, r}: 1 \leq j \leq l_{1}\right\}=0$ for every $1 \leq r \leq l_{1}$.

Proof. First, we show how to construct $z, e$, and $\sigma$ satisfying (ii) and (iii). We shall use a kind of Gauss elimination and Lemma 4. Namely, we transform the matrix $e$. To start with, we put

$$
e=\left(e_{1}, \ldots, e_{l_{1}}\right)=\left(b_{1}, \ldots, b_{l_{1}}\right) .
$$

We shall perform some ${ }^{h} A$-linear transformations of columns and permutations of rows of the matrix $e$ and replace $e$ each time by the resulting matrix. These transformations will not change the rank from the right of the family of columns of $e$. At the end, we get a matrix $e$ satisfying the required properties (ii), (iii).

We have $\operatorname{rankr}(e)=l_{1}$. If $l_{1}=0$, i.e., if $e$ is an empty matrix, then this is the end of the construction: $z$ is an empty matrix. Suppose that $l_{1}>0$. We choose indices $1 \leq i_{0} \leq k$ and $1 \leq j_{0} \leq l_{1}$ such that ord $e_{i_{0}, j_{0}}=\min _{1 \leq j \leq l_{1}}\left\{\right.$ ord $\left.e_{j}\right\}$. Permuting rows and columns of $e$, we may assume without loss of generality that $\left(i_{0}, j_{0}\right)=(1,1)$.

By Lemma 4, we get elements $w_{i, 1}, w_{i, i} \in{ }^{h} A$ of degree at most $(2 n+1) 4 d$ such that $e_{1,1} w_{1, i}=e_{1, i} w_{i, i}, 1 \leq i \leq l_{1}$, and ord $w_{i, i}=0$ for every $1 \leq i \leq l_{1}$. Set $w^{\prime}=$ $\left(-w_{1,2}, \ldots,-w_{1, l_{1}}\right)$, and let $w^{\prime \prime}=\operatorname{diag}\left(w_{2,2}, \ldots, w_{l_{1}, l_{1}}\right)$ be the diagonal matrix. Let

$$
w=\left(\begin{array}{cc}
1 & w^{\prime} \\
0 & w^{\prime \prime}
\end{array}\right)
$$

be the square matrix with $l_{1}$ rows. We replace $e$ by $e w$. Now

$$
e=\left(\begin{array}{cc}
e_{1,1} & 0 \\
E_{2,1} & E_{2,2}
\end{array}\right)
$$

where $E_{2,2}$ has $l_{1}-1$ columns, and

$$
\min _{1 \leq j \leq l_{1}}\left\{\operatorname{ord} b_{j}\right\}=\operatorname{ord} e_{1,1}=\min _{1 \leq j \leq l_{1}}\left\{\operatorname{ord} e_{j}\right\}
$$

(for the new matrix $e$ ).

We apply recursively the described construction to the matrix $E_{2,2}$ in place of $e$. So, using only linear transformations of columns with indices $2, \ldots, l_{1}$ and permutation of rows with indices $2, \ldots, k$, we transform $e$ to

$$
\sigma e \tau=\left(\begin{array}{cc}
e_{1,1} & 0 \\
E_{2,1}^{\prime} & E_{2,2}^{\prime} \\
E_{2,1}^{\prime \prime} & E_{2,2}^{\prime \prime}
\end{array}\right), \quad \tau=\left(\begin{array}{cc}
1 & 0 \\
0 & \tau^{\prime}
\end{array}\right)
$$


where $\sigma$ is a permutation matrix and $\tau^{\prime}$ is a square matrix with $l_{1}-1$ rows (it transforms $\left.E_{2,2}\right)$, the matrix $E_{2,2}^{\prime}=\operatorname{diag}\left(e_{2,2}, \ldots, e_{l_{1}, l_{1}}\right)$ is a diagonal matrix with $l_{1}-1 \geq 0$ columns, and all the elements $e_{2,2}, \ldots, e_{l_{1}, l_{1}} \in{ }^{h} A$ are nonzero. We shall assume without loss of generality that $\sigma=1$ is the identity matrix. We replace $e$ by $e \tau$. Condition (iii) is fulfilled for the resulting matrix $e$, and moreover, by (iii) applied recursively to $\left(E_{2,2}, E_{2,2}^{\prime}, E_{2,2}^{\prime \prime}\right)$ in place of $\left(e, e^{\prime}, e^{\prime \prime}\right)$, and by (33), the same equalities in (33) are satisfied for the new matrix $e$.

Let $E_{2,1}^{\prime}=\left(e_{2,1}, \ldots, e_{l_{1}, 1}\right)^{t}$, where $t$ denotes transposition. By Lemma 4 , there are nonzero elements $v_{1,1}, \ldots, v_{l_{1}, 1} \in{ }^{h} A$ of degrees at most

$$
(2 n+1)\left(\max \left\{\operatorname{deg} e_{i, j}: 1 \leq i \leq l_{1}, j=1, i\right\}+1\right) l_{1}^{2}
$$

such that $e_{i, 1} v_{1,1}=e_{i, i} v_{i, 1}$ and $\min \left\{\right.$ ord $\left.v_{1, i}: 1 \leq i \leq l_{1}\right\}=0$ for all $1 \leq i \leq l_{1}-1$. Let $v^{\prime}=\left(-v_{2,1}, \ldots,-v_{l_{1}, 1}\right)^{t}$, and let $v^{\prime \prime}$ be the identity matrix of size $l_{1}-1$. Put

$$
v=\left(\begin{array}{cc}
v_{1,1} & 0 \\
v^{\prime} & v^{\prime \prime}
\end{array}\right) .
$$

We replace $e$ by $e v$ and put $z=w \tau v$. Recall that, without loss of generality, $\sigma=1$ is the identity permutation. We have $e=\left(b_{1}, \ldots, b_{l_{1}}\right) z$. These Gauss elimination transformations of $e$ do not change the rank from the right of the family of columns of $e$. This can be proved easily by using recursion on $l$; cf. Lemma 8 below. Now the matrix $e$ satisfies the required conditions (ii) and (iii), and $\sigma=1$.

Now we change the notation. We denote the matrix $z$ obtained so far by $z^{\prime}$. Let $z^{\prime}=$ $\left(z_{1}^{\prime}, \ldots, z_{l_{1}}^{\prime}\right)$, where $z_{j}^{\prime}$ is the $j$ th column of $z^{\prime}$. Our aim now is to prove the existence of a matrix $z$ satisfying (i)-(iii). By Lemma 4 , for every $1 \leq r \leq l_{1}$, there are homogeneous elements $z_{j, r} \in{ }^{h} A, 1 \leq j \leq l_{1}$, such that $\left(z_{1, r}, \ldots, z_{l_{1}, r}\right) \neq(0, \ldots, 0), \operatorname{deg} z_{j, r}=d_{r}^{\prime}+\mu_{r}$ or $z_{j, r}=0$ for all $1 \leq j \leq l_{1}$,

$$
\sum_{1 \leq j \leq l_{1}} b_{i, j} z_{j, r}=0 \quad \text { for every } \quad 1 \leq i \leq l_{1}, i \neq r
$$

and estimates (32) for the degrees are true. Put $z=\left(z_{j, r}\right)_{1 \leq j, r \leq l_{1}}$ and $d_{r}^{\prime \prime}=-\mu_{r}$. Let $z=\left(z_{1}, \ldots, z_{l_{1}}\right)$, where $z_{j}$ is the $j$ th column of $z$. Hence, $z_{j}=\left(z_{1, j}, \ldots, z_{l_{1}, j}\right)^{t}$.

Lemma 7. For every $1 \leq r \leq l_{1}$ we have

$$
\sum_{1 \leq j \leq l_{1}} b_{r, j} z_{j, r} \neq 0
$$

and for every $1 \leq r \leq l_{1}$ there are nonzero homogeneous elements $g_{r}^{\prime}, g_{r} \in{ }^{h} A$ such that $z_{r}^{\prime} g_{r}^{\prime}=z_{r} g_{r}$.

Proof. Consider the matrix $\left(z^{\prime}, z_{r}\right)$ with $l_{1}$ rows and $l_{1}+1$ columns. Using Lemma 4 , we see that there are homogeneous elements $h_{1}, \ldots, h_{l_{1}+1} \in{ }^{h} A$ (depending on $r$ ) such that $\left(h_{1}, \ldots, h_{l_{1}+1}\right) \neq(0, \ldots, 0)$ and the following is fulfilled. Denote $h=\left(h_{1}, \ldots, h_{l_{1}+1}\right)^{t}$ and $h^{\prime}=\left(h_{1}, \ldots, h_{l_{1}}\right)^{t}$. Then

$$
z^{\prime} h^{\prime}+z_{r} h_{l_{1}+1}=0
$$

(at present, we do not need any estimate on degrees from Lemma 4; we only need the existence of $h$ ). Denote by $b^{\prime \prime}$ the submatrix formed by the first $l_{1}$ rows of the matrix $\left(b_{1}, \ldots, b_{l_{1}}\right)$. Multiplying (37) by $b^{\prime \prime}$ from the left, we get

$$
b^{\prime \prime} z^{\prime} h^{\prime}+b^{\prime \prime} z_{r} h_{l_{1}+1}=0 .
$$

But $b^{\prime \prime} z^{\prime}$ is a diagonal matrix with nonzero elements on the diagonal; see (ii) (with $z^{\prime}$ in place of $z$ ). Hence, by (35) and (38), $h_{j}=0$ for every $j \neq r$. 
Now suppose that $h_{r}=0$. Then $h^{\prime}=0$. Since $z_{r} \neq 0$, we have $h_{l_{1}+1}=0$ by (3.7). Hence, $h=(0, \ldots, 0)^{t}$, a contradiction.

Suppose that $h_{l_{1}+1}=0$. Then by (38) we have $h_{r}=0$. Hence $h=(0, \ldots, 0)^{t}$ and again we get a contradiction.

Thus, $h_{r} \neq 0$ and $h_{l_{1}+1} \neq 0$. Now (38) implies (36). Put $g_{r}^{\prime}=h_{r}$ and $g_{r}=-h_{l_{1}+1}$. We have $z_{r}^{\prime} g_{r}^{\prime}=z_{r} g_{r}$ by (37). The lemma is proved.

We return to the proof of Lemma 6 . Now (i)-(iii) are satisfied by Lemma 7 . The last assertions of Lemma 6 are proved much as those in Lemma 4. Lemma 6 is proved.

\section{$\S 7$. An ALGORITHM FOR SOLVING LINEAR SYSTEMS With COEFFICIENTS IN ${ }^{h} A$}

Let $u=\left(u_{1}, \ldots, u_{l}\right) \in{ }^{h} A^{l}$. Suppose that all nonzero $u_{j}$ are homogeneous elements of degree $-d_{j}^{\prime}+\rho$ for an integer $\rho$, and that $-d_{j}^{\prime}+\rho<d^{\prime}$ for an integer $d^{\prime}>1$. Let $b=\left(b_{i, j}\right)_{1 \leq i \leq k, 1 \leq j \leq l}$ be a matrix as in Lemma 6 , having $k$ rows and $l$ columns (but now $k$ and $l$ are arbitrary). So, $\operatorname{deg} b_{i, j}=d_{i}-d_{j}^{\prime}<d$ for all $i, j$ and $d \geq 2$. Let $Z=\left(Z_{1}, \ldots, Z_{k}\right)$ be unknowns. Consider the linear system

$$
\sum_{1 \leq i \leq k} Z_{i} b_{i, j}=u_{j}, \quad 1 \leq j \leq l,
$$

or, what is the same,

$$
Z b=u
$$

Denote

$$
\operatorname{ord} u=\min _{1 \leq i \leq l}\left\{\operatorname{ord} u_{i}\right\}
$$

Similar notation will be used for other vectors and matrices. In this section we describe an algorithm for solving linear systems over ${ }^{h} A$ and prove the following theorem for an infinite field $F$ (for a finite field $F$ this theorem reduces easily to the case where $F$ is infinite, but we shall not use this theorem for a finite field $F$ in this paper).

Theorem 2. Suppose that system (39) has a solution over ${ }^{h} A$. Then the set of all solutions of (39) over $^{h} A$ can be represented in the form

$$
J+z^{*},
$$

where $J \subset{ }^{h} A^{l}$ is an ${ }^{h} A$-submodule of all the solutions of the homogeneous system corresponding to (39) (i.e., system (39) with all $u_{j}=0$ ) and $z^{*}$ is a particular solution of (39). Moreover, the following assertions hold true.

(A) The solution $z^{*}$ can be chosen so that ord $z^{*} \geq \operatorname{ord} u-\nu$, where $\nu \geq 0$ is an integer bounded from above by $(d l)^{2^{O(n)}}$. The degree $\operatorname{deg} z^{*}$ is bounded from above by $d^{\prime}+(d l)^{2^{O(n)}}$.

(B) $J$ admits a system of generators of degrees bounded from above by $(d l)^{2^{O(n)}}$. The number of elements of this system of generators is bounded from above by $k(d l)^{2^{O(n)}}$.

The constants in $O(n)$ in (A) and (B) are absolute. Moreover, if all $b_{i, j}$ and $u_{j}$ do not depend on $X_{n}$ (i.e., they can be represented as sums of monomials that do not contain $\left.X_{n}\right)$, then $z^{*}$ and all the generators of the module $J$ also possess this property.

Proof. Let $l_{1}=\operatorname{rankr}\left(b_{1}, \ldots, b_{l}\right)$. If $l_{1}=0$, then $u=(0, \ldots, 0), J={ }^{h} A^{k}$, and we can take $z^{*}=(0, \ldots, 0)$. So, in what follows we shall assume that $l_{1}>0$. Then $1 \leq l_{1} \leq k$ by Lemma 4. Permuting equations of (39), we may assume without loss of generality that $\left(b_{1}, \ldots, b_{l_{1}}\right)$ are linearly independent from the right over ${ }^{h} A$. Let $\sigma, z, e, e^{\prime}, e^{\prime \prime}$ be the matrices occurring in Lemma 6 . As in the proof of Lemma 6 , we shall assume 
without loss of generality that $\sigma=1$. Denote by $b^{\prime}$ the submatrix of $b$ formed by the first $l_{1}$ columns of $b$, i.e., $b^{\prime}=\left(b_{1}, \ldots, b_{l_{1}}\right)$. By Lemma 4 , there are nonzero homogeneous elements $q_{1,1}, \ldots, q_{l_{1}, l_{1}}$ of degrees at most

$$
(2 n+1)\left(\max \left\{\operatorname{deg} e_{i, i}: 1 \leq i \leq l_{1}\right\}+1\right) l_{1}^{2}
$$

such that $e_{1,1} q_{1,1}=e_{i, i} q_{i, i}$ and $\min \left\{\operatorname{ord} q_{i, i}: 1 \leq i \leq l_{1}\right\}=0$. We introduce the diagonal matrix $q=\operatorname{diag}\left(q_{1,1}, \ldots, q_{l_{1}, l_{1}}\right)$. Let $\nu_{0}=$ ord $e_{1,1} q_{1,1}$. Then $\operatorname{ord}\left(b^{\prime} z q\right) \geq \nu_{0}$ by Lemma 6 (iii). Let $X_{0}^{\nu_{0}} \delta=b^{\prime} z q$. Then $\delta$ is a matrix with coefficients in ${ }^{h} A$, and

$$
\delta=\left(\delta_{i, j}\right)_{1 \leq i \leq k, 1 \leq j \leq l_{1}}=\left(\begin{array}{c}
\delta^{\prime} \\
\delta^{\prime \prime}
\end{array}\right),
$$

where $\delta^{\prime}=\operatorname{diag}\left(\delta_{1,1}, \ldots, \delta_{l_{1}, l_{1}}\right)$ is a diagonal matrix with homogeneous coefficients from ${ }^{h} A$ and such that all the elements on the diagonal are nonzero and equal, i.e., $\delta_{j, j}=\delta_{1,1}$ for every $1 \leq j \leq l_{1}$. Also, ord $\delta_{1,1}=0$. Next, $\delta^{\prime \prime}=\left(\delta_{i, j}\right)_{l_{1}+1 \leq i \leq k, 1 \leq j \leq l_{1}}$. We have $\operatorname{ord}(u z q) \geq \nu_{0}$ because, otherwise, system (39) has no solutions. Obviously, ord $u \leq$ $\operatorname{ord}(u z q)$. Denote $u^{\prime}=\left(u_{1}^{\prime}, \ldots, u_{l_{1}}^{\prime}\right)=X_{0}^{-\nu_{0}} u z q \in{ }^{h} A^{l}$. Then ord $u^{\prime} \geq \operatorname{ord}(u)-\nu_{0}$.

By Lemma 6 (i), and since $q$ is a diagonal matrix with nonzero homogeneous entries on the diagonal, there are integers $d_{j}^{\prime \prime}, 1 \leq j \leq l_{1}$, such that for all $i, j$ we have

$$
\operatorname{deg} \delta_{i, j}=d_{i}-d_{j}^{\prime \prime}
$$

or $\delta_{i, j}=0$. Besides that, for the same reason there is an integer $\rho^{\prime}$ such that $\operatorname{deg} u_{j}^{\prime}=$ $-d_{j}^{\prime \prime}+\rho^{\prime}$ or $u_{j}^{\prime}=0$ for all $1 \leq j \leq l_{1}$ (here we leave the details to the reader).

Consider the linear system

$$
Z \delta=u^{\prime}
$$

Lemma 8. Suppose that system (39) has a solution over ${ }^{h} A$. Then the linear system (42) is equivalent to (39), i.e., the sets of solutions of systems (42) and (39) over ${ }^{h} A$ coincide.

Proof. The system $Z b^{\prime} z=u z$ is equivalent to (39) by Lemma 5. System (42) is equivalent to $Z b^{\prime} z=u z$ because the ring ${ }^{h} A$ has no zero divisors. The lemma is proved.

Remark 6. We have $\operatorname{rankr}\left(b_{1}, \ldots, b_{l}\right)=l_{1}$. Hence, by Lemma 6 , for every $l_{1}+1 \leq$ $j \leq l$ there are homogeneous $z_{j, j}, z_{1, j}, \ldots, z_{l_{1}, j} \in{ }^{h} A$ such that $z_{j, j} \neq 0, b_{j} z_{j, j}+$ $\sum_{1 \leq r \leq l_{1}} b_{r} z_{r, j}=0$, and all $\operatorname{deg} z_{j, j}, \operatorname{deg} z_{r, j}$ are bounded from above by $(2 n+1)\left(l_{1}+1\right)^{2} d$. Put $u_{j}^{\prime}=u_{j} z_{j, j}+\sum_{1 \leq r \leq l_{1}} u_{r} z_{r, j}, l_{1}+1 \leq j \leq l$. Then system (39) has a solution if and only if system (42) has a solution and $u_{j}^{\prime}=0$ for all $l_{1}+1 \leq j \leq l$. This follows from Lemmas 8 and 5. But in what follows for our aims it suffices to use only Lemma 8.

Remark 7. Assume that $\operatorname{deg}_{X_{n}} b_{i, j} \leq 0$ for all $i, j$, i.e., the elements of the matrix $b$ do not depend on $X_{n}$. Then by Lemmas 4 and 6 and by our construction, all the elements of the matrices $b, z, q, \delta, \delta^{\prime}, \delta^{\prime \prime}$ also do not depend on $X_{n}$.

By Lemma 4 and Remark 3, for every $l_{1}+1 \leq i \leq k$, there are homogeneous elements $g_{i, i}, g_{i, j} \in{ }^{h} A, 1 \leq j \leq l_{1}$, such that

$$
g_{i, i} \delta_{i, j}=g_{i, j} \delta_{1,1}, \quad 1 \leq j \leq l_{1}
$$

all the degrees $\operatorname{deg} g_{i, i}, \operatorname{deg} g_{i, j}, 1 \leq j \leq l_{1}$, are bounded from above by

$$
(2 n+1)\left(l_{1}+1\right)^{2}\left(\max \left\{\operatorname{deg} \delta_{i, j}: 1 \leq j \leq k\right\}+1\right),
$$

and $\min _{1 \leq j \leq l_{1}}\left\{\right.$ ord $g_{i, i}$, ord $\left.g_{i, j}\right\}=0$. Therefore, ord $g_{i, i}=0$ for every $l_{1}+1 \leq i \leq k$, because ord $\delta_{1,1}=0$.

We need an analog of the Noether normalization theorem from commutative algebra; cf. also Lemma 3.1 in [7]. 
Lemma 9. Let $h \in{ }^{h} A$ be an arbitrary nonzero element, and let $\operatorname{deg} h=\varepsilon$. There is a linear automorphism $\alpha$ of the algebra ${ }^{h} A$,

$$
\begin{aligned}
& \alpha:{ }^{h} A \rightarrow{ }^{h} A, \quad \alpha\left(X_{i}\right)=\sum_{1 \leq j \leq n}\left(\alpha_{1, i, j} X_{j}+\alpha_{2, i, j} D_{j}\right), \\
& \alpha\left(D_{i}\right)=\sum_{1 \leq j \leq n}\left(\alpha_{3, i, j} X_{j}+\alpha_{4, i, j} D_{j}\right), \quad \alpha\left(X_{0}\right)=X_{0}, \quad 1 \leq i \leq n,
\end{aligned}
$$

such that all $\alpha_{s, i, j}$ are in $F$ and $\operatorname{deg}_{D_{n}} \alpha(h)=\varepsilon$. Moreover, one can choose $\alpha$ so that, additionally, for every $H \in{ }^{h} A$, if $\operatorname{deg}_{X_{n}} H=0$, then $\operatorname{deg}_{X_{n}} \alpha(H)=0$.

Proof. To start with, it is not difficult to construct a linear automorphism $\beta$ such that $\beta\left(X_{0}\right)=X_{0}, \beta\left(X_{n}\right)=X_{n}, \beta\left(D_{n}\right)=D_{n}$,

$$
\beta\left(X_{i}\right)=\beta_{1, i} X_{i}+\beta_{2, i} D_{i}, \quad \beta\left(D_{i}\right)=\beta_{3, i} X_{i}+\beta_{4, i} D_{i}, \quad 1 \leq i \leq n,
$$

and $\beta(h)$ contains a monomial $a_{i_{1}, \ldots, i_{n}} D_{1}^{i_{1}}, \ldots, D_{n}^{i_{n}}$ with $a_{i_{1}, \ldots, i_{n}} \neq 0$ and $i_{1}+\cdots+i_{n}=\varepsilon$, i.e., $\varepsilon=\operatorname{deg}_{D_{1}, \ldots, D_{n}} \beta(h)$. After that, we can find an automorphism $\gamma$ such that $\gamma\left(X_{0}\right)=$ $X_{0}$,

$$
\begin{aligned}
\gamma\left(X_{i}\right) & =X_{i}, \quad \gamma\left(D_{i}\right)=D_{i}+\gamma_{i} D_{n}, \quad 1 \leq i \leq n-1, \\
\gamma\left(X_{n}\right) & =X_{n}-\sum_{1 \leq i \leq n-1} \gamma_{i} X_{i}, \gamma\left(D_{n}\right)=D_{n},
\end{aligned}
$$

where $\gamma_{i} \in F$ for all $1 \leq i \leq n-1$, and $(\gamma \circ \beta)(h)$ contains a monomial $a D_{n}^{\varepsilon}$ with $0 \neq a \in F$. Put $\alpha=\gamma \circ \beta$. Obviously, if $H \in{ }^{h} A$ and $\operatorname{deg}_{X_{n}} H=0$, then $\operatorname{deg}_{X_{n}} \alpha(H)=0$. The lemma is proved.

Put $h=\delta_{1,1} g_{l_{1}+1, l_{1}+1} g_{l_{1}+2, l_{1}+2} \cdots g_{k, k}$. Then $h \in{ }^{h} A$ is a nonzero homogeneous element and ord $h=0$. By Lemma 9, we obtain an automorphism $\alpha$. Applying $\alpha$ to the coefficients of system (42), we get a new linear system. Again by Lemma 9, if all the coefficients of system (42) do not depend on $X_{n}$, then all the coefficients of the new system also do not depend on $X_{n}$. In what follows, to simplify the notation, we shall assume without loss of generality that $\alpha=1$. Thus, $h$ contains a monomial $a D_{n}^{\varepsilon}$ with $0 \neq a \in F$, where $\varepsilon=\operatorname{deg} h$. Then

$$
\operatorname{deg}_{D_{n}} \delta_{1,1}=\operatorname{deg} \delta_{1,1}, \quad \operatorname{deg}_{D_{n}} g_{i, i}=\operatorname{deg} g_{i, i}, \quad l_{1}+1 \leq i \leq k .
$$

Let $z=\left(z_{1}, \ldots, z_{k}\right) \in{ }^{h} A^{k}$ be a solution of (42). Then (43) implies that we can uniquely represent $z_{i}$ in the form

$$
z_{i}=z_{i}^{\prime} g_{i, i}+\sum_{0 \leq r<\operatorname{deg} g_{i, i}} z_{i, r} D_{n}^{r}, \quad l_{1}+1 \leq i \leq k,
$$

where $z_{i}^{\prime}, z_{i, r} \in{ }^{h} A$, and $\operatorname{deg}_{D_{n}} z_{i, r} \leq 0$ for all $l_{1}+1 \leq i \leq k, 0 \leq r<\operatorname{deg}_{D_{1}} g_{i, i}$. Again by (43), we can uniquely represent $u_{j}^{\prime}$ in the form

$$
u_{j}^{\prime}=u_{j}^{\prime \prime} \delta_{1,1}+\sum_{0 \leq s<\operatorname{deg} \delta_{1,1}} u_{j, s}^{\prime} D_{n}^{s}, \quad 1 \leq j \leq l_{1},
$$

where $u_{j}^{\prime \prime}, u_{j, s}^{\prime} \in{ }^{h} A$, and $\operatorname{deg}_{D_{n}} u_{j, s}^{\prime} \leq 0$ for all $1 \leq j \leq l_{1}, 0 \leq s<\operatorname{deg}_{D_{1}} g_{i, i}$. Finally, by (43), for all $l_{1}+1 \leq i \leq k, 1 \leq j \leq l_{1}$, and $0 \leq r<\operatorname{deg}_{D_{1}} g_{i, i}$, there is a unique representation

$$
D_{n}^{r} \delta_{i, j}=\delta_{i, r, j} \delta_{1,1}+\sum_{0 \leq s<\operatorname{deg} \delta_{1,1}} \delta_{i, r, j, s} D_{n}^{s}
$$


where $\delta_{i, r, j}, \delta_{i, r, j, s} \in{ }^{h} A$, and $\operatorname{deg}_{D_{n}} \delta_{i, r, j, s} \leq 0$ for all the $i, r, j, s$ involved. Put

$$
\begin{aligned}
& \mathcal{I}=\left\{(i, r): l_{1}+1 \leq i \leq k \& 0 \leq r<\operatorname{deg} g_{i, i}\right\}, \\
& \mathcal{J}=\left\{(j, s): 1 \leq j \leq l_{1} \& 1 \leq s<\operatorname{deg} \delta_{1,1}\right\} .
\end{aligned}
$$

Therefore,

$$
\begin{aligned}
& z_{j}=-\sum_{l_{1}+1 \leq i \leq k} z_{i}^{\prime} g_{i, j}-\sum_{(i, r) \in \mathcal{I}} z_{i, r} \delta_{i, r, j}+u_{j}^{\prime \prime}, \quad 1 \leq j \leq l_{1}, \\
& \sum_{(i, r) \in \mathcal{I}} z_{i, r} \delta_{i, r, j, s}=u_{j, s}^{\prime}, \quad(j, s) \in \mathcal{J} .
\end{aligned}
$$

We introduce new unknowns $Z_{i, r},(i, r) \in \mathcal{I}$. By (44)-(46), system (39) reduces to the linear system

$$
\sum_{(i, r) \in \mathcal{I}} Z_{i, r} \delta_{i, r, j, s}=u_{j, s}^{\prime}, \quad(j, s) \in \mathcal{J} .
$$

More precisely, any solution of system (39) is given by (44), (45), where the $z_{i}^{\prime} \in{ }^{h} A$ are arbitrary and $z_{i, r}$ is a solution of system (46) over ${ }^{h} A$ (we emphasize that this solution $z_{i, r}$ may depend on $D_{n}$, although we can restrict ourselves to solutions $z_{i, r}$ that do not depend on $D_{n}$ ). Note that all $\delta_{i, r, j, s}$ and $u_{j, s}^{\prime}$ are homogeneous elements of ${ }^{h} A$. Put $d_{i, r}=d_{i}+r$, $(i, r) \in \mathcal{I}$ and $d_{j, s}^{\prime}=d_{j}^{\prime \prime}+s,(j, s) \in \mathcal{J}, \widetilde{\rho}=\rho^{\prime}$, where $d_{j}, d_{i}^{\prime \prime}, \rho^{\prime}$ are as introduced above; see (41). Then $\operatorname{deg} \delta_{i, r, j, s}=d_{i, r}-d_{j, s}^{\prime}$ or $\delta_{i, r, j, s}=0$, and $\operatorname{deg} u_{j, s}^{\prime}=-d_{j, s}^{\prime}+\widetilde{\rho}$ or $u_{j, s}^{\prime}=0$, for all $(i, r) \in \mathcal{I},(j, s) \in \mathcal{J}$. This follows immediately from our construction (we leave the details to the reader).

Now all the coefficients of system (47) do not depend on $D_{n}$. As we have proved, if the coefficients of (39) do not depend on $X_{n}$, then the coefficients of (47) also do not depend on $X_{n}$, and hence, in this case they do not depend on $X_{n}, D_{n}$.

If the coefficients of (47) depend on $X_{n}$, we perform an automorphism $X_{n} \mapsto D_{n}$ $D_{n} \mapsto-X_{n}, X_{i} \mapsto X_{i}, D_{i} \mapsto D_{i}, 1 \leq i \leq n-1$. Now the coefficients of system (47) do not depend on $X_{n}$ (but depend on $D_{n}$ ).

After that, we apply our construction recursively to system (47). Here, we need to lean upon Remark 5 , because the integers $d_{j, s}^{\prime}$ are not necessarily positive.

More precisely, denote ${ }^{h} A=A^{(n)}$ for brevity. As the input of the step in question we have system (39) over the ring $A^{(n)}$. Now, as the input of the next recursive step we have system (47) over $A^{\left(n^{\prime}\right)}$, where $n^{\prime}=n$ if at least one of the coefficients of system (39) depends on $X_{n}$, and $n^{\prime}=n-1$ if all the coefficients of system (39) do not depend on $X_{n}$ (thus, $n$ is replaced by $n^{\prime}$, and it reduces after each two steps of recursion; $n^{\prime}$ is a new value of $n$ for the input of the recursive step). Let $J^{\prime}$ be the module of solutions of the homogeneous system corresponding to (47) over the ring $A^{\left(n^{\prime}\right)}$. Then, obviously, ${ }^{h} A J^{\prime}$ is the module of solutions of the same homogeneous system over ${ }^{h} A$. Each system of generators of $J^{\prime}$ over $A^{\left(n^{\prime}\right)}$ is a system of generators of ${ }^{h} A J^{\prime}$ over ${ }^{h} A$. Similarly, a particular solution of system (47) over $A^{\left(n^{\prime}\right)}$ is a particular solution of system (47) over ${ }^{h} A$.

At the final step of the recursion, at least one of the following conditions is fulfilled:

- $l_{1}=0$ for the newly obtained system (in place of (39));

- $n=0$ (although in the statement of the theorem we have $n \geq 1$, see $\S 1$, we are interested only in Weyl algebras).

If $l_{1}=0$, we get the required $z^{*}$ and $J$ at this recursive step immediately; see above. If $n=0$, then $\mathcal{I}=\mathcal{J}=\varnothing$. Hence, using (45) for $n=0$, we get the required $z^{*}$ and $J$ for $n=0$. 
Thus, using the recursive assumption, we get a particular solution $Z_{i, r}=z_{i, r}^{*},(i, r) \in$ $\mathcal{I}$, of system (47), an integer $\nu_{1}$ (in place of $\nu$ from assertion (A)) satisfying the inequality

$$
\min _{(i, r) \in \mathcal{I}}\left\{\operatorname{ord} z_{i, r}^{*}\right\} \geq \min _{(j, s) \in \mathcal{J}}\left\{\operatorname{ord} u_{j, s}^{\prime}\right\}-\nu_{1},
$$

and a system of generators

$$
\left(z_{\alpha, i, r}\right)_{(i, r) \in \mathcal{I}}, \quad 1 \leq \alpha \leq \beta,
$$

of the module $J^{\prime}$ of solutions of the homogeneous system corresponding to (47). Put

$$
\begin{aligned}
z_{j}^{*} & =-\sum_{(i, r) \in \mathcal{I}} z_{i, r}^{*} \delta_{i, r, j}+u_{j}^{\prime \prime}, \quad 1 \leq j \leq l_{1}, \\
z_{i}^{*} & =\sum_{0 \leq r<\operatorname{deg} g_{i, i}} z_{i, r}^{*} D_{n}^{r}, \quad l_{1}+1 \leq i \leq k, \\
z^{*} & =\left(z_{1}^{*}, \ldots, z_{k}^{*}\right) .
\end{aligned}
$$

Then $z^{*}$ is a particular solution of (39). Put

$$
\begin{aligned}
z_{\alpha, j} & =-\sum_{(i, r) \in \mathcal{I}} z_{\alpha, i, r} \delta_{i, r, j}, \quad 1 \leq j \leq l_{1}, 1 \leq \alpha \leq \beta, \\
z_{\alpha, i} & =\sum_{0 \leq s<\operatorname{deg} g_{i, i}} z_{\alpha, i, s} D_{n}^{s}, \quad l_{1}+1 \leq i \leq k, \quad 1 \leq \alpha \leq \beta, \\
z_{\beta-l_{1}+i, j} & =0, \quad l_{1}+1 \leq i, j \leq k, j \neq i, \\
z_{\beta-l_{1}+i, i} & =g_{i, i}, \quad l_{1}+1 \leq i \leq k, \\
z_{\beta-l_{1}+i, j} & =-g_{i, j}, \quad 1 \leq j \leq l_{1}, \quad l_{1}+1 \leq i \leq k .
\end{aligned}
$$

Then $J=\sum_{1 \leq \alpha \leq \beta+k-l_{1}}{ }^{h} A\left(z_{\alpha, 1}, \ldots, z_{\alpha, k}\right)$. Hence, $\left(z_{\alpha, 1}, \ldots, z_{\alpha, k}\right), 1 \leq \alpha \leq \beta+k-l_{1}$, is a system of generators of the module $J$. By (48) and the definitions of $u^{\prime}, u_{j}^{\prime \prime}$, and $u_{j, s}^{\prime}$, we have ord $z^{*} \geq \operatorname{ord}(u)-\nu_{0}-\nu_{1}$. Put $\nu=\nu_{0}+\nu_{1}$.

Lemma 10. All the degrees $\operatorname{deg} \delta_{i, j}, \operatorname{deg} g_{i, i}, \operatorname{deg} g_{i, j}, \operatorname{deg} \delta_{i, r, j}, \operatorname{deg} \delta_{i, r, j, s}$ and the number $\nu_{0}$, see above, are bounded from above by $(n l d)^{O(1)}$; the degrees $\operatorname{deg} u_{j}^{\prime}$, $\operatorname{deg} u_{j}^{\prime \prime}$, $\operatorname{deg} u_{j, s}^{\prime}$ are bounded from above by $d^{\prime}+(n l d)^{O(1)}$. Next, ord $u_{j}^{\prime}$, ord $u_{j}^{\prime \prime}$, and ord $u_{j, s}^{\prime}$ are bounded from below by ord $u-\nu_{0}$. Finally, in system (47) the number $\# \mathcal{J}$ of equations is bounded from above by $(n l d)^{O(1)}$, and the number $\# \mathcal{I}$ of unknowns is bounded from above by $k(n l d)^{O(1)}$.

Proof. This follows immediately from the construction.

We return to the proof of Theorem 2. Applying Lemma 10 and, recursively, assertions (A) and (B) for the formulas giving $z^{*}$ and $J$, we get (A) and (B) from the statement of the theorem. The last claim (related to the case where all $b_{i, j}$ and $u_{j}$ do not depend on $D_{n}$ ) has already been proved. The theorem is proved.

\section{§8. Proof of Theorem 1 for Weyl algebras}

We start with showing that it suffices to prove the theorem for an infinite field $F$. Indeed, let $F_{1}$ be an infinite field such that $F_{1} \supset F$. Let $f_{1}, \ldots, f_{m}$ be a Janet basis of the module $I \otimes_{F} F_{1}$ with all the degrees $\operatorname{deg} f_{w}, 1 \leq w \leq m$, bounded from above by $d^{2^{O(n)}}$. There is a finite extension $F_{2} \supset F$ such that for all $v, i, j$ and all $1 \leq w \leq m$ the coefficient in $f_{w}$ of the monomial $e_{v, i, j}$ belongs to the field $F_{2}$. Let $a_{\alpha}, 1 \leq \alpha \leq \mu$, be the basis of the field $F_{2}$ over $F$. Then we can write $f_{w}=\sum_{1 \leq \alpha \leq \mu} a_{\alpha} f_{\alpha, w}$, where all $f_{\alpha, w}$ belong to $I$. Now $\operatorname{deg} f_{\alpha, w} \leq \operatorname{deg} f_{w}$ and $f_{\alpha, w}, 1 \leq w \leq m, 1 \leq \alpha \leq \mu$, is a Janet basis of 
the module $I$. Moreover, the reduced Janet basis of the module $I$ remains the same after an arbitrary extension of scalars. The required assertion is proved. Thus, extending the ground field $F$, we may assume without loss of generality that $F$ is infinite.

Let $a$ be a matrix as in $\S 1$. There is no loss of generality in assuming that the vectors $\left(a_{i, 1}, \ldots, a_{i, l}\right), 1 \leq i \leq k$, are linearly independent over $F$. We have $\operatorname{deg} a_{i, j}<d$. This implies $k \leq l\left(\begin{array}{c}d+2 n \\ 2 n\end{array}\right)$.

Put $b={ }^{h} a$. We define graded submodules of ${ }^{h} I$ :

$$
\begin{aligned}
& J_{0}={ }^{h} A\left(b_{1,1}, \ldots, b_{1, l}\right)+\cdots+{ }^{h} A\left(b_{k, 1}, \ldots, b_{k, l}\right), \\
& J_{\gamma}=J_{0}:\left(X_{0}^{\gamma}\right)=\left\{z \in{ }^{h} A^{l}: z X_{0}^{\gamma} \in J_{0}\right\}, \quad \gamma \geq 1 .
\end{aligned}
$$

We have the following exact sequence of graded ${ }^{h} A$-modules:

$$
{ }^{h} A^{k} \rightarrow J_{0} \rightarrow 0 .
$$

Next, we have $J_{\gamma} \subset J_{\gamma+1} \subset{ }^{h} I$ for every $\gamma \geq 0$, and ${ }^{h} I=\bigcup_{\gamma \geq 0} J_{\gamma}$. Since ${ }^{h} A$ is Noetherian, there exists $N \geq 0$ such that ${ }^{h} I=J_{N}$. Therefore, to construct a system of generators of ${ }^{h} I$, it suffices to compute the smallest $N$ such that ${ }^{h} I=J_{N}$ and to find a system of generators of $J_{N}$.

Lemma 11. ${ }^{h} I=J_{N}$ for some $N$ bounded from above by $(d l)^{2^{O(n)}}$. There is a system of generators $b_{1}, \ldots, b_{s}$ of the module $J_{N}$ such that $s$ and all the degrees $\operatorname{deg} b_{v}, 1 \leq v \leq s$, are bounded from above by $(d l)^{2^{O(n)}}$.

Proof. We show that $J_{N+1} \subset J_{N}$ for $N \geq \nu$. Let $u \in J_{N+1}$. Consider system (39). By assertion (A) of Theorem 2, there is a particular solution $z^{*}$ of (39) such that ord $z^{*} \geq 1$. Hence, $u \in X_{0} J_{N} \subset J_{N}$. The claim is proved. Thus, ${ }^{h} I=J_{\nu}$.

We replace $\left(u_{1}, \ldots, u_{l}\right)$ in $(39)$ by $\left(U_{1} X_{0}^{\nu}, \ldots, U_{l} X_{0}^{\nu}\right)$, where $U_{1}, \ldots, U_{l}$ are new unknowns. Then, applying statement (B) of Theorem 2 to this new homogeneous linear system with respect to the unknowns $U_{1}, \ldots, U_{l}, Z_{1}, \ldots, Z_{k}$, we get the required estimates for the number of generators of $J_{\nu}$ and for the degrees of these generators. The lemma is proved.

Corollary 1. Let $\left(a_{i, 1}, \ldots, a_{i, l}\right), 1 \leq i \leq l$, be as at the beginning of the section, and let the integer $N$ be as in Lemma 11. Then, for every integer $m \geq 0$, the F-linear space

$$
A_{m+N}\left(a_{1,1}, \ldots, a_{1, l}\right)+\cdots+A_{m+N}\left(a_{k, 1}, \ldots, a_{k, l}\right) \text { includes } I_{m} .
$$

Proof. By Lemma 11, we have $\left(J_{0}\right)_{m+N} \supset X_{0}^{N}\left(J_{N}\right)_{m}=X_{0}^{N}\left({ }^{h} I\right)_{m}$. Taking the affine parts yields (50). The corollary is proved.

Now everything is ready for the proof of Theorem 1. By Lemmas 11 and 1, there is a system of generators of the module $\operatorname{gr}(I)$ with degrees bounded from above by $(d l)^{2^{O(n)}}$. By Lemma 12 (see Appendix 1), the Hilbert function $H(\operatorname{gr}(I), m)$ is stable for $m \geq$ $(d l)^{2^{O(n)}}$. By (11) (see $\S 2$ ), the Hilbert function $H(I, m)$ is stable for all $m \geq(d l)^{2^{O(n)}}$.

Consider the linear order $<$ on the monomials in ${ }^{h} A^{l}$ that is induced by the linear order $<$ on the monomials in $A^{l}$; see $\S 4$. Then the monomial (i.e., generated by monomials) submodule ${ }^{c} I \subset{ }^{c} A^{l}$ is well defined, see $\S 4$, where ${ }^{c} A=F\left[X_{0}, \ldots, X_{n}, D_{1}, \ldots, D_{n}\right]$ is the polynomial ring. By $(24)$, the Hilbert function $H\left({ }^{c} I, m\right)$ is stable for all $m \geq(d l)^{2^{O(n)}}$. Hence, all the coefficients of the Hilbert polynomial of ${ }^{c} I$ are bounded from above by $(d l)^{2^{O(n)}}$. Therefore, by Lemma 13 , the module ${ }^{C} I$ has a system of generators with degrees $(d l)^{2^{O(n)}}$. We can assume without loss of generality that this system of generators of ${ }^{c} I$ consists of monomials. The sets of monomials in ${ }^{c} I$ and in $\operatorname{Hdt}\left({ }^{h} I\right)$ are in a natural degree-preserving one-to-one correspondence; see $\S 4$. Therefore, see $\S 4$, the degrees of 
all the elements of a Janet basis of ${ }^{h} I$ with respect to the induced linear order $<$ are bounded from above by $(d l)^{2^{O(n)}}$. Since the ideal ${ }^{h} I$ is homogeneous, the same bound is valid for the degrees of all the elements (they are homogeneous) of the reduced Janet basis of ${ }^{h} I$. Hence, by Lemma 3 (iii) (see $\S 4$ ), the same is true for some Janet basis $f_{1}, \ldots, f_{m}$ (respectively, by Lemma 3 (ii), for the reduced Janet basis in the case where the initial order $<$ is degree-compatible) of the module $I$ with respect to the linear order $<$ on the monomials in $A^{l}$.

It remains to consider the case where $l=1$ and an admissible linear order $<$ is arbitrary. We need to obtain estimates for the reduced Janet basis of $I$ in this case. In the case in question, the linear order $<$ is given on the set of pairs of multi-indices $(i, j), i, j \in \mathbb{Z}_{+}^{n}$. Now (see, e.g., [13, p. 58]), there is a real ordered field $R$ and a linear form $L \in R\left[Y_{1}, \ldots, Y_{n}, Z_{1}, \ldots, Z_{n}\right]$ with positive coefficients such that, for all pairs $(i, j)$, $\left(i^{\prime}, j^{\prime}\right)$ of multi-indices, $\left(i^{\prime}, j^{\prime}\right)<(i, j)$ if and only if

$$
L\left(i-i^{\prime}, j-j^{\prime}\right)=L\left(i_{1}-i_{1}^{\prime}, \ldots, i_{n}-i_{n}^{\prime}, j_{1}-j_{1}^{\prime}, \ldots, j_{n}-j_{n}^{\prime}\right)>0
$$

in the real ordered field $R$.

Let $\psi_{1}<\cdots<\psi_{a}$ be all the monomials in $X_{1}, \ldots, X_{n}, D_{1}, \ldots, D_{n}$ with nonzero coefficients in the elements $f_{1}, \ldots, f_{m}$, and let $\left(i^{(1)}, j^{(1)}\right)<\cdots<\left(i^{(a)}, j^{(a)}\right)$ be the corresponding pairs of multi-indices. Let $\varepsilon>0$ be an infinitesimal with respect to the field $R$. Now

$$
L\left(i^{(s+1)}-i^{(s)}, j^{(s+1)}-j^{(s)}\right) \geq \varepsilon, \quad 1 \leq s \leq a-1,
$$

in the field $R(\varepsilon)$. Let $U=\sum_{1 \leq w \leq n}\left(u_{w} Y_{w}+v_{w} Z_{w}\right)$ be a generic linear form in the variables $Y_{1}, \ldots, Y_{n}, Z_{1}, \ldots, Z_{n}$; i.e., the family $\left\{u_{w}, v_{w}\right\}_{1 \leq w \leq n}$ of coefficients of $U$ has the transcendency degree $2 n$ over $R(\varepsilon)$. Consider the following system of linear inequalities with coefficients in $\mathbb{Q}[\varepsilon]$ with respect to $u_{w}, v_{w}, 1 \leq w \leq n$,

$$
\begin{cases}U\left(i^{(s+1)}-i^{(s)}, j^{(s+1)}-j^{(s)}\right) \geq \varepsilon, & 1 \leq s \leq a-1 \\ u_{w} \geq \varepsilon, & 1 \leq w \leq n \\ v_{w} \geq \varepsilon, & 1 \leq w \leq n\end{cases}
$$

Let $K_{\varepsilon}$ be the set of solutions of system (52) in $R(\varepsilon)^{2 n}$. By (51), and since all the coefficients of the linear form $L$ are positive, system (52) has a solution in $R(\varepsilon)^{2 n}$. The left-hand sides of the inequalities in (52) are linear forms in $u_{w}, v_{w}, 1 \leq w \leq n$, with integral coefficients. We denote them by $Q_{1}, \ldots, Q_{\mu}, \mu=a-1+2 n$. Observe that the absolute values of the coefficients of the linear forms $Q_{1}, \ldots, Q_{\mu}$ are bounded from above by $d^{2^{O(n)}}$.

We show that there are indices $1 \leq w_{1}<\cdots<w_{s} \leq \mu$ and $s \leq 2 n$ such that $\mathcal{Z}\left(Q_{w_{1}}-\varepsilon, \ldots, Q_{w_{s}}-\varepsilon\right) \subset K_{\varepsilon}$ (here $\mathcal{Z}\left(Q_{w_{1}}-\varepsilon, \ldots, Q_{w_{s}}-\varepsilon\right)$ is the set of all common zeros of the polynomials $Q_{w_{1}}-\varepsilon, \ldots, Q_{w_{s}}-\varepsilon$ in $\left.R(\varepsilon)^{2 n}\right)$ and the linear forms $Q_{w_{1}}, \ldots, Q_{w_{s}}$ are linearly independent over $\mathbb{Q}$. Indeed, we can construct $Q_{w_{1}}, \ldots, Q_{w_{s}}$ recursively, by choosing subsequently $Q_{w_{\alpha}}, \alpha \geq 1$, such that $\mathcal{Z}\left(Q_{w_{\alpha}}-\varepsilon\right)$ has a nonempty intersection with the boundary of $\mathcal{Z}\left(Q_{w_{1}}-\varepsilon, \ldots, Q_{w_{\alpha-1}}-\varepsilon\right) \cap K_{\varepsilon}$ (we leave the details to the reader).

Solving the linear system $Q_{w_{1}}-\varepsilon=\cdots=Q_{w_{s}}-\varepsilon=0$, we see that there is a point $\left(u_{w}^{\prime}, v_{w}^{\prime}\right)_{1 \leq w \leq n} \in K_{\varepsilon}$ such that $u_{w}^{\prime}=a_{w} \varepsilon / c$ and $v_{w}^{\prime}=b_{w} \varepsilon / c$, where all $a_{w}, b_{w}, c$ are positive integers with absolute values bounded from above by $d^{2^{O(n)}}$. Put $\varepsilon^{*}=1$, $u_{w}^{*}=a_{w} / c$ and $v_{w}^{*}=b_{w} / c, 1 \leq w \leq n$. We view (52) as a linear system with respect to all $u_{w}, v_{w}$ and $\varepsilon$. Then $u_{w}^{*}, v_{w}^{*}$ and $\varepsilon^{*}>0$ is a solution of $(52)$ in $\mathbb{Q}^{2 n+1}$. Set $L^{*}=c \sum_{1 \leq w \leq n}\left(u_{w}^{*} Y_{w}+v_{w}^{*} Z_{w}\right)$. 
Now $L^{*} \in \mathbb{Z}\left[Y_{1}, \ldots, Y_{n}, Z_{1}, \ldots, Z_{n}\right]$ is a linear form with positive integral coefficients bounded from above by $d^{2^{O(n)}}$ and such that

$$
L^{*}\left(i^{(s+1)}-i^{(s)}, j^{(s+1)}-j^{(s)}\right)>0, \quad 1 \leq s \leq a-1 .
$$

We assume without loss of generality that $\operatorname{Hdt}\left(f_{1}\right), \ldots, \operatorname{Hdt}\left(f_{m}\right)$ is the family of leading monomials of the reduced Janet basis $f_{1}^{\prime}, \ldots, f_{m}^{\prime}$ of the module $I$ with respect to the linear order $<$, and $\operatorname{Hdt}\left(f_{1}\right)>\cdots>\operatorname{Hdt}\left(f_{m}\right)$. For any $g \in A$, put $\lambda(g)=L^{*}(i, j)$, where $\operatorname{Hdt}(g)=g_{i, j} X^{i} D^{j}, 0 \neq g_{i, j} \in F$. Then inequality (53) and the definitions show that $\lambda\left(f_{w}\right)=\lambda\left(f_{w}^{\prime}\right)$ for all $1 \leq w \leq m$. Hence, all $\lambda\left(f_{w}^{\prime}\right)$ are bounded from above by $d^{2^{O(n)}}$. But, obviously, $\operatorname{deg} f_{w}^{\prime} \leq \lambda\left(f_{w}^{\prime}\right), 1 \leq w \leq m$. Theorem 1 is proved for Weyl algebras.

\section{§9. The case of an algebra of Differential operators}

Extending the ground field $F$, we may suppose without loss of generality that the field $F$ is infinite. We denote by $B=F\left(X_{1}, \ldots, X_{n}\right)\left[D_{1}, \ldots, D_{n}\right]$ the algebra of differential operators. Recall that $A \subset B$, so that relations (2) are satisfied. Next, each element $f \in B$ can be uniquely represented in the form

$$
f=\sum_{j_{1}, \ldots, j_{n} \geq 0} f_{j_{1}, \ldots, j_{n}} D_{1}^{j_{1}} \cdots D_{n}^{j_{n}}=\sum_{j \in \mathbb{Z}_{+}^{n}} f_{j} D^{j},
$$

where all $f_{j_{1}, \ldots, j_{n}}=f_{j}$ belong to $F\left(X_{1}, \ldots, X_{n}\right)$ and $F\left(X_{1}, \ldots, X_{n}\right)$ is the field of rational functions over $F$. Everywhere in $\S \S 1$ and 2 , we replace $A, X^{i} D^{j}, \operatorname{deg} f=$ $\operatorname{deg}_{X_{1}, \ldots, X_{n}, D_{1}, \ldots, D_{n}} f, \operatorname{dim}_{F} M, e_{v, i, j}, f_{v, i, j} \in F$, and $(v, i, j),(i, j),\left(i^{\prime}, j^{\prime}\right),\left(i^{\prime \prime}, j^{\prime \prime}\right)$ by $B$, $D^{j}, \operatorname{deg} f=\operatorname{deg}_{D_{1}, \ldots, D_{n}} f, \operatorname{dim}_{F\left(X_{1}, \ldots, X_{n}\right)} M, e_{v, j}, f_{v, j} \in F\left(X_{1}, \ldots, X_{n}\right)$, and $(v, j), j$, $j^{\prime}, j^{\prime \prime}$, respectively. This leads to the definition of the Janet basis and all other objects occurring in $\S 1$ for the case of the algebra of differential operators.

The definition of the homogenization ${ }^{h} B$ of $B$ is similar to that of ${ }^{h} A$; see $\S 3$. Namely, ${ }^{h} B=F\left(X_{1}, \ldots, X_{n}\right)\left[X_{0}, D_{1}, \ldots, D_{n}\right]$ is given by the relations

$$
\begin{aligned}
X_{i} X_{j} & =X_{j} X_{i}, D_{i} D_{j}=D_{j} D_{i}, \quad \text { for all } i, j, \\
D_{i} X_{i}-X_{i} D_{i} & =X_{0}, \quad 1 \leq i \leq n, \quad X_{i} D_{j}=D_{j} X_{i} \quad \text { for all } i \neq j .
\end{aligned}
$$

The further considerations are similar to the case of the Weyl algebra $A$ with minor changes. We leave them to the reader. For example, Theorem 2 for the case of the algebra of differential operators is the same. One need only to replace $A,{ }^{h} A$, and $X_{n}$ by $B,{ }^{h} B$ and $D_{n}$ everywhere, respectively. Thus, Theorem 1 can be proved in the case where $A$ is an algebra of differential operators (but now it is $B$ ). Theorem 1 is proved completely.

One can consider a more general algebra of differential operators. Let $\mathcal{F}$ be a field with $n$ derivatives $D_{1}, \ldots, D_{n}$. Then $K_{n}=\mathcal{F}\left[D_{1}, \ldots, D_{n}\right]$ is an algebra of differential operators, and its homogenization ${ }^{h} K_{n}$ can be defind as before, by means of adding a variable $X_{0}$ satisfying the relations

$$
D_{i} D_{j}=D_{j} D_{i}, \quad X_{0} D_{i}=D_{i} X_{0}, \quad D_{i} f-f D_{i}=f_{D_{i}} X_{0}
$$

for all $i, j$ and all elements $f \in \mathcal{F}$, where $f_{D_{i}} \in \mathcal{F}$ denotes the result of an application of $D_{i}$ to $f$. Following the proof of Theorem 1, we can deduce the statement below.

Remark 8. Bounds similar to those in Theorem 1 hold true for $K_{n}$ (in place of the algebra of differential operators $A$ ). 


\section{Appendix 1: Degrees of Generators of A Graded module OVER A POLYNOMIAL RING, AND ITS HILBERT FUNCTION}

We give a short proof of the following result; cf. [1, 12, 6, 4]. Let $\mathcal{A}=F\left[X_{0}, \ldots, X_{n}\right]$ be a graded polynomial ring. The homogeneous elements of $\mathcal{A}$ are homogeneous polynomials in $X_{0}, \ldots, X_{n}$.

Lemma 12. Let $I \subset \mathcal{A}^{l}$ be a graded $\mathcal{A}$-module with a system of generators $f_{1}, \ldots, f_{m}$ of degrees less than $d$, where $d \geq 2$. Then the Hilbert function $H\left(\mathcal{A}^{l} / I, m\right)=\operatorname{dim}_{F}\left(\mathcal{A}^{l} / I\right)_{m}$ is stable for $m \geq(d l)^{2^{O(n+1)}}$. Furthermore, all the coefficients of the Hilbert polynomial of $\mathcal{A}^{l} / I$ are bounded from above by $(d l)^{2^{O(n+1)}}$.

Proof. Extending the ground field $F$, we may suppose without loss of generality that the field $F$ is infinite. Denote $M=\mathcal{A}^{l} / I$. Let $L \in F\left[X_{0}, \ldots, X_{n}\right]$ be a linear form in general position. Let $K$ stand for the kernel of the morphism $M \rightarrow M$ of multiplication by $L$. We have $K=\left\{z \in \mathcal{A}^{l}: L z=\sum_{1 \leq i \leq m} f_{i} z_{i}, z_{i} \in \mathcal{A}\right\}$. Hence, solving a linear system over $\mathcal{A}$, we see that $K$ has a system of generators $g_{1}, \ldots, g_{\mu}$ with degrees bounded from above by $(d l)^{2^{O(n+1)}}$. Let $\mathfrak{P}$ be an arbitrary associated prime ideal of the module $M$ such that $\mathfrak{P} \neq\left(X_{0}, \ldots, X_{n}\right)$. Since $L$ is in general position, we have $L \notin \mathfrak{P}$. Therefore, $\mathfrak{P}$ is not an associated prime ideal of $K$. Consequently, $K_{N}=0$ for all sufficiently large $N$. So, $X_{i}^{N} g_{j} \in I$ for sufficiently large $N$ and all $i, j$. Hence, $g_{j}=\sum_{1 \leq i \leq m} y_{j, i} f_{i}$, where $y_{j, i} \in$ $F\left(X_{i}\right)\left[X_{0}, \ldots, X_{n}\right]$. Solving a linear system over the $\operatorname{ring} F\left(X_{i}\right)\left[X_{0}, \ldots, X_{n}\right]$, we get a bound on the denominators from $F\left[X_{i}\right]$ of all $y_{j, i}$. Since all $g_{j}$ and $f_{i}$ are homogeneous, we may assume without loss of generality that all the denominators are $X_{i}^{N}$. Thus, we get an upper bound for $N$. Namely, $N$ is bounded from above by $(d l)^{2^{O(n+1)}}$.

Therefore, the sequence

$$
0 \rightarrow M_{m} \rightarrow M_{m+1} \rightarrow(M / L M)_{m+1} \rightarrow 0
$$

is exact for $m \geq(d l)^{2^{O(n+1)}}$. But $M / L M=\mathcal{A}^{l} /\left(I+L \mathcal{A}^{l}\right)$ is a module over a polynomial ring $F\left[X_{0}, \ldots, X_{n}\right] /(L) \simeq F\left[X_{0}, \ldots, X_{n-1}\right]$. Hence, by the inductive assumption, the Hilbert function $H\left(\mathcal{A}^{l} /\left(I+L \mathcal{A}^{l}\right), m\right)$ is stable for $m \geq(d l)^{2^{O(n)}}$. Now (55) implies that the Hilbert function $H\left(\mathcal{A}^{l} / I, m\right)$ is stable for $m \geq(d l)^{2^{O(n+1)}}$.

Obviously, for $m<(d l)^{2^{O(n+1)}}$ the values $H\left(\mathcal{A}^{l} / I, m\right)$ are bounded from above by $(d l)^{2^{O(n+1)}}$. Using the Newton interpolation, we conclude that all the coefficients of the Hilbert polynomial of $\mathcal{A}^{l} / I$ are bounded from above by $(d l)^{2^{O(n+1)}}$. The lemma is proved.

We also need a converse to Lemma 12 .

Lemma 13. Let $I \subset \mathcal{A}^{l}$ be a graded $\mathcal{A}$-module. Assume that the Hilbert function $H\left(\mathcal{A}^{l} / I, m\right)=\operatorname{dim}_{F}\left(\mathcal{A}^{l} / I\right)_{m}$ is stable for $m \geq D$ and that all absolute values of the coefficients of the Hilbert polynomial of the module $\mathcal{A}^{l} / I$ are bounded from above by $D$, for some integer $D>1$. Then I has a system of generators $f_{1}, \ldots, f_{m}$ with degrees $D^{2^{O(n+1)}}$.

Proof. Let $f_{1}, \ldots, f_{m}$ be the reduced Gröbner basis of $I$ with respect to an admissible linear order $<$ on the monomials in $\mathcal{A}^{l}$; cf. the definitions in $\S \S 1$ and 4 . The degree of a monomial from $\mathcal{A}^{l}$ is defined as in $\S \S 1$ and 4 . We assume additionally that the linear order under consideration is degree-compatible; i.e., for any two monomials $z_{1}, z_{2}$, if $\operatorname{deg} z_{1}<$ $\operatorname{deg} z_{2}$, then $z_{1}<z_{2}$. For every $z \in \mathcal{A}^{l}$, the greatest monomial $\operatorname{Hdt}(z)$ is $\operatorname{defined}$. The monomial module $\operatorname{Hdt}(I)$ is generated by all $\operatorname{Hdt}(z), z \in I$. Now, $\operatorname{Hdt}\left(f_{1}\right), \ldots, \operatorname{Hdt}\left(f_{m}\right)$ is a minimal system of generators of $\operatorname{Hdt}(I)$, and $\operatorname{deg} f_{i}=\operatorname{deg} \operatorname{Hdt}\left(f_{i}\right)$ for every $1 \leq i \leq m$. The values of the Hilbert functions $H\left(\mathcal{A}^{l} / \operatorname{Hdt}(I), m\right)=H\left(\mathcal{A}^{l} / I, m\right)$ coincide for all 
$m \geq 0$; cf. $\S 4$. Thus, replacing $I$ by $\operatorname{Hdt}(I)$, we shall assume in what follows in this proof that $I$ is a monomial module.

For every $1 \leq i \leq l$, denote by $\mathcal{A}_{i} \subset \mathcal{A}^{l}$ the $i$ th direct summand of $\mathcal{A}^{l}$. Put $I_{i}=I \cap \mathcal{A}_{i}$, $1 \leq i \leq l$. Then $I \simeq \bigoplus_{1<i<l} I_{i}$ because $I$ is a monomial module. Next, for every $1 \leq \alpha \leq m$ there is $1 \leq i \leq l$ such that $f_{\alpha} \in I_{i}$. We identify $\mathcal{A}_{i}=\mathcal{A}$. Then $I_{i} \subset \mathcal{A}$ is a homogeneous monomial ideal. The case where $I_{i}=\mathcal{A}$ for some $i$ is not excluded. For Hilbert functions, we have

$$
H\left(\mathcal{A}^{l} / I, m\right)=\sum_{1 \leq i \leq l} H\left(\mathcal{A} / I_{i}, m\right), \quad m \geq 0 .
$$

If $\left(\mathcal{A} / I_{i}\right)_{D}=0$ for some $i$, then $\left(\mathcal{A} / I_{i}\right)_{m}=0$ for every $m \geq D$. In this case the ideal $I_{i}$ is generated by $\sum_{0 \leq m \leq D}\left(I_{i}\right)_{m}$. Hence for $m \geq D$ we can omit this index $i$ in the sum on the right in (56). Therefore, in this case the proof reduces to a smaller $l$. So, we may assume without loss of generality that $\left(\mathcal{A} / I_{i}\right)_{D} \neq 0,1 \leq i \leq l$. Next, we use an exact description of the Hilbert function of a homogeneous ideal; see [4, §7]. Namely, there are unique integers $b_{i, 0} \geq b_{i, 1} \geq \cdots \geq b_{i, n+2}=0$ such that

$$
H\left(\mathcal{A} / I_{i}, m\right)=\left(\begin{array}{c}
m+n+1 \\
n+1
\end{array}\right)-1-\sum_{1 \leq j \leq n+1}\left(\begin{array}{c}
m-b_{i, j}+j-1 \\
j
\end{array}\right)
$$

for all sufficiently large $m$ and

$$
b_{i, 0}=\min \left\{d: d \geq b_{i, 1} \text { and for all } m \geq d,(57) \text { is true }\right\} .
$$

This description (without the constants $b_{i, 0}$ ) dates back to the classical paper [11. The integers $b_{i, 0}, \ldots, b_{i, n+2}$ are called the Macaulay constants of the ideal $I_{i}$. We have

$$
h(i, m)=H\left(\mathcal{A} / I_{i}, m\right)-\left(\begin{array}{c}
m+n+1 \\
n+1
\end{array}\right)+1+\sum_{1 \leq j \leq n+1}\left(\begin{array}{c}
m-b_{i, j}+j-1 \\
j
\end{array}\right) \geq 0
$$

for every $m \geq b_{i, 1}$; see [4, $\S 7$ ]. By Lemma 7.2 in [4], for all $1 \leq \alpha \leq m$, if $f_{\alpha} \in I_{i}$, then $\operatorname{deg} f_{\alpha} \leq b_{i, 0}$. Hence, it suffices to prove that all $b_{i, 0}, 1 \leq i \leq l$, are bounded from above by $D^{2^{O(n+1)}}$.

By (56) and (57), the coefficient of $m^{n-j}, 0 \leq j \leq n$, in the Hilbert polynomial of $\mathcal{A}^{l} / I$ is

$$
\frac{\mu_{j}}{(n+1-j) !} \sum_{1 \leq i \leq l} b_{i, n+1-j}+\sum_{0 \leq v \leq j-1} \sum_{1 \leq i \leq l} \frac{1}{(n+1-v) !} \mu_{j, v}\left(b_{i, n+1-v}\right),
$$

where $0 \neq \mu_{j}$ is an integer, and $\mu_{j, v} \in \mathbb{Z}[Z], 0 \leq v \leq j-1$, is a polynomial with integral coefficients and $\operatorname{deg} \mu_{j, v}=j-v+1$. Moreover, $\left|\mu_{j}\right|$ and the absolute values of the coefficients of all the polynomials $\mu_{j, v}$ are bounded from above by, say, $2^{O\left(n^{2}\right)}$. Denote $b_{j}=\sum_{1 \leq i \leq l} b_{i, j}, 0 \leq j \leq n+2$. By the condition of the lemma, the coefficients of the Hilbert polynomial of $\mathcal{A}^{l} / I$ are bounded from above by $D$. Hence, via (60), we can recursively estimate $b_{n+1}, b_{n}, \ldots, b_{1}$. Namely, $b_{n+1-j}=\left(2^{n^{2}} l D\right)^{2^{O(j+1)}}, 0 \leq j \leq n$. Consequently, $b_{1}=(l D)^{2^{O(n+1)}}$. Observe that $b_{i, 1} \leq \max _{1 \leq i \leq l} b_{i, 1} \leq b_{1}$ for every $1 \leq i \leq m$.

Now, let $m \geq \max _{1 \leq i \leq l} b_{i, 1}$. By (59), if $h(i, m) \neq 0$ for some $1 \leq i \leq l$, then $m<D$; i.e., $m$ is less than the bound $D$ for the stabilization of the Hilbert function of $\mathcal{A}^{l} / I$. Thus, $b_{i, 0} \leq \max \left\{b_{i, 1}, D\right\}$ by (58). Hence, $b_{i, 0}$ is bounded from above by $(l D)^{2^{O(n+1)}}$.

We have $\left(\mathcal{A} / I_{i}\right)_{D} \neq 0$ for every $1 \leq i \leq l$. This implies $H\left(\mathcal{A}^{l} / I, D\right) \geq l$. Let $c_{j}$ denote the $j$ th coefficient of the Hilbert polynomial of the module $\mathcal{A}^{l} / I$. Now $\left|c_{j}\right| D^{j} \geq l /(n+1)$ for at least one $j$. Hence, $D^{n+1}(n+1) \geq l$ by the condition of the lemma. This implies that $l^{2^{O(n+1)}}$ is bounded from above by $D^{2^{O(n+1)}}$. Therefore, $b_{i, 0}$ is bounded from above by $D^{2^{O(n+1)}}$. The lemma is proved. 


\section{Appendix 2: Bound For the Gröbner BASIS OF A MONOMIAL MOdule IN TERMS OF THE COEFFICIENTS OF ITS HILBERT POLYNOMIAL}

We denote by $C_{l}=\mathbb{Z}_{+}^{n} \cup \cdots \cup \mathbb{Z}_{+}^{n}$ the disjoint union of $l$ copies of the semigroup $\mathbb{Z}_{+}^{n}=\left\{\left(i_{1}, \ldots, i_{n}\right) \in \mathbb{Z}^{n}: i_{j} \geq 0,1 \leq j \leq n\right\}$. A subset of $C_{l}$ that intersects each disjoint copy of $\mathbb{Z}_{+}^{n}$ by a semigroup closed with respect to addition in $\mathbb{Z}_{+}^{n}$ is called an ideal of $C_{l}$. Clearly, $I$ corresponds to a monomial submodule $M_{I}$ in the free module $\left(F\left[X_{1}, \ldots, X_{n}\right]\right)^{l}$. Any ideal $I$ in $C_{l}$ has a unique finite Gröbner basis $V=V_{I}$ corresponding to the Gröbner basis of $M_{I}$. Denote $T=C_{l} \backslash I$. The degree of an element $u=\left(k ; i_{1}, \ldots, i_{n}\right) \in C_{l}, 1 \leq$ $k \leq l$, is defined as $|u|=i_{1}+\cdots+i_{n}$. The degree of a subset in $C_{l}$ is defined as the supremum of the degrees of its elements. The Hilbert function $H_{T}(z)$ is equal to the number of vectors $u \in T$ such that $|u| \leq z$. Hence, $H_{T}(z)=\sum_{0 \leq s \leq m} c_{s} z^{s}, z \geq z_{0}$, for a suitable $z_{0}$ and integers $c_{0}, \ldots, c_{m}$, where $m \leq n$. Let $c=\max _{0 \leq s \leq m}\left|c_{s}\right| s !+1$.

Proposition 1 (cf. [6, 12, 4]). The degree of $V$ does not exceed $(\mathrm{cn})^{2^{O(m)}}$.

Proof. By an $s$-cone, $0 \leq s \leq n$, we shall mean a subset of the $k$ th copy of $\mathbb{Z}_{+}^{n}$ in $C_{l}$ for some $1 \leq k \leq l$ of the form

$$
P=\left\{X_{j_{1}}=i_{1}, \ldots, X_{j_{n-s}}=i_{n-s}\right\}
$$

for suitable $1 \leq j_{1}, \ldots, j_{n-s} \leq n$. We define the degree of the $s$-cone $(61)$ as $|P|=$ $i_{1}+\cdots+i_{n-s}$ (note that this definition is different from that in [4]). By a predecessor of (61) we mean each $s$-cone in the same $k$ th copy of $\mathbb{Z}_{+}^{n}$ of the type

$$
\left\{X_{j_{1}}=i_{1}, \ldots, X_{j_{p-1}}=i_{p-1}, X_{j_{p}}=i_{p}-1, X_{j_{p+1}}=i_{p+1}, \ldots, X_{j_{n-s}}=i_{n-s}\right\}
$$

for some $1 \leq p \leq n-s$, provided that $i_{p} \geq 1$. We fix an arbitrary linear order on $s$-cones compatible with the predecessor relation.

Using inverse recursion on $s$, we gradually fill $T$ (as a union) by $s$-cones with $0 \leq s \leq m$. We start with $s=m$. Assume that a current union $T_{0} \subset T$ of $m$-cones is already constructed (at the very beginning we put $T_{0}=\varnothing$ ) and that an $m$-cone of the form (61) with $s=m$ is the smallest one (with respect to the fixed linear order on $m$-cones) that is contained in $T$ and not contained in $T_{0}$. Observe that each predecessor of this $m$-cone was added to $T_{0}$ at earlier steps of its construction. Since the total number of $m$-cones added to $T_{0}$ does not exceed $c_{m} m !<c$, we see that the degree of every such $m$-cone is less than $c_{m} m$ ! (we use the fact that the first $m$-cone added to $T_{0}$ has degree 0 ).

For the recursive step, assume that the current $T_{0}$ is a union of all possible $m$-cones, $(m-1)$-cones, $\ldots,(s+1)$-cones and perhaps, some $s$-cones. This can be expressed as $\operatorname{deg}\left(H_{T}-H_{T_{0}}\right) \leq s$. Again, as in the base, we take the smallest $s$-cone of the form (61) that is contained in $T$ and not contained in $T_{0}$. Observe that each predecessor of the type (62) of this $s$-cone is contained in an appropriate $r$-cone $Q, r \geq s$, such that $Q$ was added to $T_{0}$ at earlier steps of its construction and $Q \subset\left\{X_{j_{p}}=i_{p}-1\right\}$. Hence,

$$
|Q| \geq i_{p}-1 \text {. }
$$

This construction terminates when $T_{0}=T$. We denote by $t_{s}$ the number of $s$-cones added to $T_{0}$ and by $k_{s}$ the maximum of their degrees. We have already seen that $t_{m}, k_{m}<c$.

Now, we use inverse induction on $s$ to prove that $t_{s}, k_{s} \leq(\mathrm{cn})^{2^{O(m-s)}}$. For this, we introduce a special semilattice on the set of cones. Let $\mathcal{C}=\left\{C_{\alpha, \beta}\right\}_{\alpha, \beta}, \quad 0 \leq \beta \leq \gamma_{\alpha}$, be a family of cones of the form (61), where $\operatorname{dim} C_{\alpha, \beta}=\alpha$. By an $\alpha$-piece we call an $\alpha$-cone that is the intersection of some cones in $\mathcal{C}$. All the pieces constitute a semilattice $\mathcal{L}$ with respect to intersection with the maximal elements in $\mathcal{C}$. We treat $\mathcal{L}$ also as a partially ordered set with respect to inclusion. Clearly, the depth of $\mathcal{L}$ is at most $n+1$. Our nearest purpose is to estimate the size of $\mathcal{L}$ from above. To simplify the bound, 
we assume (and this will suffice for our goal in the sequel) that $\gamma_{\alpha} \leq(\mathrm{cn})^{2^{O(m-\alpha)}}$ for $s \leq \alpha \leq m$ and $\gamma_{\alpha}=0$ when $\alpha<s$, although in the general case the required bound can be obtained in the same way. Moreover, we assume that the constant in $O(\ldots)$ is sufficiently large. In what follows all the constants in $O(\ldots)$ coincide.

Lemma 14. Suppose that $\gamma_{\alpha} \leq(c n)^{2^{O(m-\alpha)}}$ for all $s \leq \alpha \leq m$; see above. Then the number of $\alpha$-pieces in $\mathcal{L}$ does not exceed $(c n)^{2^{O(m-\alpha)}+1}$ for $s \leq \alpha \leq m$, or $(c n)^{2^{O(m-s)}(s-\alpha+1)+1}$ for $\alpha<s$.

Proof. For each $\alpha$-piece, we choose its arbitrary irredundant representation as the intersection of cones in $\mathcal{C}$. Let $\delta$ be the minimal dimension of those cones. Then this intersection contains at most $\delta-\alpha+1$ cones. Therefore, the number of possible $\alpha$-pieces does not exceed

which proves the lemma.

$$
\sum_{\max \{\alpha, s\} \leq \delta \leq m}(c n)^{2^{O(m-\delta)}(\delta-\alpha+1)},
$$

Now we return to estimating $t_{s}, k_{s}$ by inverse induction on $s$. In the construction described above, let the current $T_{0}$ be the union of all added $m$-cones, $(m-1)$-cones, $\ldots$, $s$-cones. We denote this family of cones by $\mathcal{C}$ and consider the corresponding semilattice $\mathcal{L}$ (see above). Our next purpose is to represent $T_{0}$ as a $\mathbb{Z}$-linear combination of pieces in $\mathcal{L}$ via a kind of the inclusion-exclusion formula. We assign the coefficients of this combination by recursion in $\mathcal{L}$. As a base, we assign 1 to each maximal piece, i.e., the elements of $\mathcal{C}$. At a recursive step, if for some piece $P \in \mathcal{L}$ the coefficients are already assigned to all the pieces greater than $P$, then we assign to $P$ the coefficient $\epsilon_{P}$ in such a way that the sum of the coefficients assigned to $P$ and to all greater pieces equals 1 . Therefore,

$$
T_{0}=\sum_{P \in \mathcal{L}} \epsilon_{P} P
$$

where the sum is understood in the sense of multisets. Consequently,

$$
H_{T_{0}}(z)=\sum_{P \in \mathcal{L}} \epsilon_{P}\left(\begin{array}{c}
z-|P|+\operatorname{dim} P \\
\operatorname{dim} P
\end{array}\right)
$$

for sufficiently large $z$. We recall that $\operatorname{deg}\left(H_{T}-H_{T_{0}}\right) \leq s-1$.

Now we estimate the coefficients $\left|\epsilon_{P}\right|$ with the help of induction in the semilattice $\mathcal{L}$. The inductive hypothesis on $t_{\alpha} \leq(c n)^{2^{O(m-\alpha)}}, s \leq \alpha \leq m$, and Lemma 14 imply that

$$
\sum_{\operatorname{dim} P=\lambda}\left|\epsilon_{P}\right| \leq(c n)^{2^{O(m-\lambda)}}, \quad s-1 \leq \lambda \leq m
$$

in accordance with inverse induction on $\lambda$ and the definition of $\epsilon_{P}$. In fact, one could estimate also $\sum_{\operatorname{dim} P=\lambda}\left|\epsilon_{P}\right|$ in a similar way when $\lambda<s-1$, but we do not need this. The inductive hypothesis on $k_{\alpha} \leq(c n)^{2^{O(m-\alpha)}}, s \leq \alpha \leq m$, and (64) imply that the coefficient in $H_{T_{0}}(z)$ of the power $z^{\alpha}$ does not exceed $(c n)^{2^{O(m-\alpha)}}, s-1 \leq \alpha \leq m$ (actually, by the inequality $\operatorname{deg}\left(H_{T}-H_{T_{0}}\right) \leq s-1$, the coefficients of the powers $z^{\alpha}$ for $s \leq \alpha \leq m$ are less than $c$ ). In particular, the coefficient of the power $z^{s-1}$ does not exceed $(c n)^{2^{O(m-s+1)}}$. Denote $H_{T}-H_{T_{0}}=\eta z^{s-1}+\cdots$. When constructing $T_{0}$, we add $(s-1)$-cones to it $t_{s-1}=\eta(s-1)$ ! times. Hence, $t_{s-1} \leq(c n)^{2^{O(m-s+1)}}$. This justifies the inductive step for $t_{s-1}$.

We prove that $k_{s-1} \leq(c n)^{2^{O(m-s+1)}}$. We observe that, for each $(s-1)$-cone $P$ added to $T_{0}$, either every one of its predecessors is contained in a cone of dimension at least $s$, or some predecessor is an $(s-1)$-cone. In the former case, $|P| \leq\left(\max _{s \leq \alpha \leq m} k_{\alpha}+1\right)(n-s+1)$ 
(by (63)), while in the latter case, $|P|$ is greater by 1 than the degree of that predecessor. Thus, $k_{s-1} \leq\left(\max _{s \leq \alpha \leq m} k_{\alpha}+1\right)(n-s+1)+t_{s-1}$. Finally, we use the inductive hypothesis for $k_{m}, \ldots, k_{s}$ and the inequality on $t_{s-1}$ obtained above.

To complete the proof of the proposition, it suffices to observe that for any vector in the basis $V$ treated as a 0-cone, each of its predecessors of the form (62) for $s=0$ is included in an appropriate $r$-cone occurring in the above construction, whence the degree of $V$ does not exceed $\left(\max _{0 \leq \alpha \leq m} k_{\alpha}+1\right) n$, again by (63) (cf. above).

\section{ACKNOWLEDGMENT}

The authors are grateful to the Max-Planck Institut für Mathematik, Bonn for its hospitality during the stay when this paper was written.

\section{REFERENCES}

[1] D. A. Bayer, The division algorithm and the Hilbert scheme, Ph.D. Thesis, Harvard, 1982.

[2] A. Chistov and D. Grigor'ev, Complexity of quantifier elimination in the theory of algebraically closed fields, Mathematical Foundations of Computer Science (Prague, 1984), Lecture Notes in Comput. Sci., vol. 176, Springer, Berlin, 1984, pp. 17-31. MR0783435 (86i:03040)

[3] D. Cox, J. Little, and D. O'Shea, Using algebraic geometry, Grad. Texts in Math., vol. 185, SpringerVerlag, New York, 1998. MR1639811(99h:13033)

[4] T. Dubé, The structure of polynomial ideals and Gröbner bases, SIAM J. Comput. 19 (1990), 750-775. MR 1053942 (91h:13021)

[5] A. Galligo, Some algorithmic questions on ideals of differential operators, EUROCAL' 85, Vol. 2 (Linz, 1985), Lecture Notes in Comput. Sci., vol. 204, Springer, Berlin, 1985, pp. 413-421. MR:0826576 (87g:32012)

[6] M. Giusti, Some effectivity problems in polynomial ideal theory, EUROSAM 84 (Cambridge, 1984), Lecture Notes in Comput. Sci., vol. 174, Springer, Berlin, 1984, pp. 159-171. MR0779123 (86d:12001)

[7] D. Grigoriev, Weak Bézout inequality for D-modules, J. Complexity 21 (2005), 532-542. MR 2152720 (2006b:16036)

[8] G. Hermann, Die Frage der endlich vielen Schritte in der Theorie der Polynomideale, Math. Ann. 95 (1926), 736-788. MR1512302

[9] M. Janet, Les modules de formes algébriques et la théorie générale des systèmes différentiels, Ann. Sci. École Norm. Sup. (3) 41 (1924), 27-65. MR:1509256

[10] H. Li, Noncommutative Gröbner bases and filtered-graded transfer, Lecture Notes in Math., vol. 1795, Springer-Verlag, Berlin, 2002. MR1947291 (2003i:16065)

[11] F. S. Macaulay, Some properties of enumeration in the theory of modular systems, Proc. London Math. Soc. 26 (1927), 531-555.

[12] H. Möller and F. Mora, Upper and lower bounds for the degree of Groebner bases, EUROSAM 84 (Cambridge, 1984), Lecture Notes in Comput. Sci., vol. 174, Springer, Berlin, 1984, pp. 172-183. MR0779124 (86k:13008)

[13] G. Ritter and V. Weispfenning, On the number of term orders, Appl. Algebra Engrg. Comm. Comput. 2 (1991), 55-79. MR.1209244 (94e:06011)

[14] F. Schwarz, Janet bases for symmetry groups, Gröbner Bases and Applications (Linz, 1998), London Math. Soc. Lecture Note Ser., vol. 251, Cambridge Univ. Press, Cambridge, 1998, pp. 221-234. MR.1708880 (2002j:35015)

[15] C. K. Yap, A new lower bound construction for commutative Thue systems with applications, J. Symbolic Comput. 12 (1991), 1-27. MR.1124303 (92i:03046)

CNRS, IRMAR, Université de Rennes Beaulieu, 35042, Rennes, France

E-mail address: dmitry.grigoryev@univ-rennes1.fr

$U R L:$ http://perso.univ-rennes1.fr/dmitry.grigoryev

Steklov Institute of Mathematics, Fontanka 27, St. Petersburg 191023, Russia

E-mail address: alch@pdmi.ras.ru

Received 30/MAR/2007

Translated by THE AUTHORS 